\title{
FLUPS: A FOURIER-BASED LIBRARY OF UNBOUNDED POISSON SOLVERS *
}

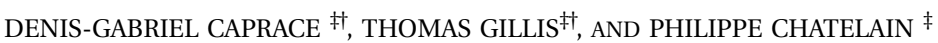

\begin{abstract}
A Fourier-based Library of Unbounded Poisson Solvers (FLUPS) for 2D and 3D homogeneous distributed grids is presented. It is designed to handle every possible combination of periodic, symmetric, semi-unbounded and fully unbounded boundary conditions for the Poisson equation on rectangular domains with uniform resolution. FLUPS leverages a dedicated implementation of 3D Fourier transforms to solve the Poisson equation using Green's functions, in a fast and memory-efficient way. Several Green's functions are available, optionally with explicit regularization, spectral truncation, or using lattice Green's functions, and provide verified convergence orders from 2 to spectral-like. The algorithm depends on the FFTW library to perform 1D transforms, while Message Passing Interface (MPI) communications enable the required remapping of data in memory. For the latter operation, a first available implementation resorts to the standard all-to-all routines. A second implementation, featuring non-blocking and persistent point-to-point communications, is however shown to be more efficient in a majority of cases and especially while taking advantage of the shared memory parallelism with OpenMP. The scalability of the algorithm, aimed at massively parallel architectures, is demonstrated up to 73720 cores. The results obtained with three different supercomputers show that the weak efficiency remains above $40 \%$ and the strong efficiency above $30 \%$ when the number of cores is multiplied by 16 , for typical problems. These figures are slightly better than those expected from a third party 3D Fast Fourier Transform (FFT) tool, with which a $20 \%$ longer execution time was also measured on average. From the outside, the solving procedure is fully automated so that the user benefits from the optimal performances while not having to handle the complexity associated with memory management, data mapping and Fourier transform computation.

The parallel code is available under Apache license 2.0 at github.com/vortexlab-uclouvain/flups.
\end{abstract}

Key word. Poisson equation, parallel computing, 3D Fourier transform, elliptic problem, free-space boundary

AMS subject classifications. 35J05,35J08,35J15,35J25,68N01

1. Introduction. The solution of Poisson problems is ubiquitous in computational physics as it concerns problems ranging from fluid dynamics and electromagnetism to particle physics. Consequently, there is a widespread need for dedicated, adapted numerical solvers. When considering time-dependent applications such as fluid dynamics for example, this equation has to be solved many times per simulation, and the efficiency of solvers is hence also crucial. In this work, we consider the Poisson equation in a 3D computational domain,

$$
\nabla^{2} \phi=f .
$$

Often such problems are unbounded in nature, as they describe a potential field induced by sources located in a compact region of space. Nevertheless it is also common to exploit symmetries that may arise from the physical configuration of the domain, or periodicity, for the sake of modeling. In all cases, the geometry of the problem naturally drives the conditions that $f$ and $\phi$ need to satisfy at the computational domain boundaries. Therefore, (1.1) has to be complemented with a set of appropriate boundary conditions (BCs) which, in each direction, specify the periodicity or an arbitrary combination of symmetries and (semi-)infinite direction.

Historically, three main families of approaches have emerged to efficiently solve (1.1): the Multigrid-based (MG) approaches [32], the Fast Multipole Methods (FMM) [17] and the Fast Fourier Transform (FFT)-based techniques [21]. In addition to the brief summary that we provide hereafter, we refer the reader to [12] and references therein for a detailed description and comparison of these approaches. In the MG approaches, (1.1) is treated as a linear system usually emerging from a finite-difference or a finitevolume discretization, where several levels of resolution are recursively used to converge towards the solution. In the FMM and the FFT-based techniques, Green's functions are employed to obtain the solution of (1.1), $\phi=G * f$, as the convolution of the right-hand side (RHS), $f$, with a precomputed kernel, $G$. The FMM treats near and far interactions separately, whereas the FFT framework does not require the distinction. Because of their specificities, the choice of the most adapted approach for a given problem is mostly driven by three criteria: (i) the computational complexity; (ii) the characteristics of the source term, $f$, and its support; and (iii) the desired BCs.

In terms of computational complexity, for a domain with $N_{p}$ sources, tree-based algorithms (like the FMM) theoretically reduce the computational cost from $\mathscr{O}\left(N_{p}^{2}\right)$ (direct interactions) down to $\mathscr{O}\left(N_{p}\right)$ [15]. Similarly, when the sources are uniformly distributed on a grid of size $N^{3}$, the multigrid approach reaches a linear complexity, $\mathscr{O}\left(N^{3}\right)$ (in 3D), where $N$ is the number of unknowns in each direction. However, in those conditions, higher computational performances can be expected from an FFTbased algorithm which allows one to cast the convolution into a pointwise multiplication in the spectral space,

$$
\hat{\phi}=\hat{G} \hat{f},
$$

where $\hat{.}$ denotes the 3D Fourier transform. The required forward and backward FFTs thus entail fast executions, in spite of their complexity of $\mathscr{O}\left(N^{3} \log (N)\right)$ operations per direction. Moreover, the three approaches can be compared in terms of their relative computational intensity [22]. While the multigrid methods present the lowest intensity, the FMM-based approaches are the most intense methods, as the computation of the multipole expansions requires many operations for fewer memory accesses.

\footnotetext{
*Submitted to the editors December 5, 2019. Corresponding authors: denis-gabriel.caprace@uclouvain.be, thomas.gillis@uclouvain.be.

Copyright: Denis-Gabriel Caprace, Thomas Gillis, Philippe Chatelain, 2019.

${ }^{\dagger}$ These authors contributed equally to the work.

${ }^{\ddagger}$ Institute of Mechanics, Materials and Civil Engineering, Université catholique de Louvain, 1348 Louvain-la-Neuve, Belgium.
} 
Most importantly, one must also consider the adequacy of the method for the source term and the geometry of its support. When $f$ is made of a distributed collection of compact sources, the FMM is most suited. On the other hand, FFT-based techniques, which require the use of a uniform grid, are best-in-class to handle evenly distributed and compactly supported source terms.

An additional advantage of the FMM over the others is that it naturally enables unbounded solutions. The spectral decomposition inherent to the Fourier transforms implies assumptions on the boundary conditions: the discreet Fourier transform (DFT) imposes periodic conditions, whereas all the combinations of odd and even symmetries are accessible through the various types of cosine and sine transforms (DCT and DST). Still, fully unbounded directions can also be achieved in an FFT-based framework using zero-padding, as described in the domain doubling technique proposed by Hockney and Eastwood [20].

In the present work, we focus on the case of source terms compactly and homogeneously supported within a parallelepipedlike domain (see $[2,7,11,28]$ for application examples). In that specific case, a uniform Cartesian mesh is well adapted and the FFT-based methods were reported to exhibit the shortest time-to-solution [12]. We here aim to present the efficient implementation of an FFT-based method, within an inclusive computational framework supporting various types of BCs.

The use of Green's functions in a discrete physical domain implies a second classification among the potential methods. In order to obtain the Green's function value on the grid, a first approach consists in sampling the Green's function corresponding to the continuous Poisson problem. In that case, a specific treatment is required to handle the singularity of the Green's functions (which can thus not be evaluated in one or several locations). In this respect, three major techniques have been proposed:

I. the replacement of the singular values with a cell-averaged equivalent [4];

II. the use of an explicit (high-order, spectral in the limit) regularization of the Green's function which thus solves the regularized Poisson problem [18, 19, 30];

III. the truncation of the Green's function in the physical space to a sphere of radius $R$ encompassing the source term. This truncated kernel method [33] leads to an analytical kernel which yields spectral-accurate results for isotropic problems. However, as detailed in [16], its generalization to anisotropic domains comes at the price of oversampling in the Fourier space, hence leading to a significant overhead, or the loss of the analytical expression.

A second approach consists in determining the fundamental solution of the discrete version of (1.1), obtained using finite differences. The corresponding Lattice Green's Functions (LGFs, hereafter denoted type (IV) ) were initially proposed in 2D [14, 25] and further applied in 3D [24]. The kernel computation involves the evaluation of an integral in infinite domain for close interactions, and requires the use of an expansion for further interactions [25]. This splitting renders the LGF also naturally well suited for an implementation of the FMM approach. In that respect, let us highlight that the computation of the interactions can be accelerated through the use of FFTs, as proposed in [24], hence providing a hybrid implementation of the FMM, optionally also with multiresolution [9].

Solving the Poisson equation is a computationally intensive task and many previous efforts have focused on parallel performance optimization, a challenge due to the inherent elliptic character of the problem. Because the distribution of the computation entails a subdivision of the domain, communications are needed between all the computational entities, which drive the overall computational performance of the algorithm. While FFT-based algorithms may rely on highly optimized (mostly serial) libraries, such as FFTW [10], their parallel 3D implementation for distributed architectures requires handling dedicated communication patterns (several of them are reviewed in [31]), which are subject to specific optimization. Among the numerous initiatives tackling 3D FFT computations, the renowned P3DFFT library [26] takes advantage of the message passing interface (MPI) and the pencil (1D) decomposition of the computational domain. As highlighted in [29], the 1D pencil decomposition indeed waives some partition-size limitations on massively parallel architectures. A pencil-based transform is able to exploit $N^{2}$ cores at most (for a cubic domain of $N^{3}$ unknowns), but requires the transposition of data, which can be efficiently implemented as in [3]. By contrast, a transform that relies on a 2D slab decomposition would be limited to the use of $N$ cores. Moreover, when used for 3D transforms, the slab decomposition features one actual all-to-all communication as the slabs need to be transformed into pencils in the third direction: one MPI rank will thus communicate with all the $N_{\text {rank }}$ other ranks. With the pencil decomposition, the communication can be arranged such that one rank only communicates twice with $\sqrt{N_{\text {rank }}}$ other ranks, which will reduce the communication latency on very large partitions. The same ideas were exploited in other packages such as PFFT [27], FFTK [6] and MPI4PYFFT [8] (see also references therein), with small variations and improvements on the parallel implementation. For the above-mentioned solutions, the scalability of the algorithm was tested on Bluegene and Cray XT/XC architectures, for some up to $64 \mathrm{k}$ cores and more, often showing similar performances. Concurrently, hardware acceleration applied to Poisson's problem has also been considered [23].

Although the above-mentioned efforts have brought several substantial advances to the solving methodology and to its numerical implementation (specifically related to 3D FFTs), an open-source solution dedicated to solving Poisson's equation with any type of BCs on a parallelepiped and uniform Cartesian mesh, that would encompass all these improvements, is still missing to date. In particular, one might wish for a solution that would hide the complexity of the FFT-based implementation from the user-whose focus should be on the boundary value problem only—but that would still benefit from the above improvements. Furthermore, such an integrated solution would push the optimization of the whole algorithm one step further, by tailoring the management of memory and data to the Poisson problem on a given mesh configuration, and by advantageously sharing the computational resources over the whole computational process.

Moreover, techniques to address certain combinations of BCs are also missing. For instance, one would be interested in 
computing the solution of (1.1) in a domain which is unbounded on one side, and symmetric on the other side. This corresponds to semi-infinite problems such as the boundary layer on a flat plate in fluid mechanics. The treatment of such a configuration in a FFT-based Poisson solver was never exposed.

This work presents the concepts that have supported the development of the Fourier-based Library of Unbounded Poisson Solvers (FLUPS). FLUPS provides an integrated solution for a variety of Poisson's problems with high computational throughput. The library, written in C++ with an API in C, supports any arbitrary combination of BCs on 2D and 3D uniform grids. The parallel implementation is based on the MPI standard and can also take advantage of shared-memory parallelism with OpenMP, depending on the size of the problem and the computational resources available. FLUPS is thus intended as an open-source, state-of-theart, and highly optimized implementation of a Poisson solver over uniform grids, available at github.com/vortexlab-uclouvain/ flups.

In this article, the general scheme for solving Poisson's equation in 3D with Green's functions is first explained. Details are given about the various types of BCs, and the consequences in the algorithm in terms of Green's functions and FFT computations. In particular, a novel method for the treatment of a semi-infinite direction with symmetry at one end is proposed. Then, a verification and validation of FLUPS is presented, focusing on the accuracy of the Poisson solver, and on the weak and strong scalability of the algorithm up to 73720 processors, measured on three different massively parallel architectures.

2. Methodology of FLUPS in 3D. FLUPS is capable of handling scalar and vector fields and, by default, assumes that data are given in a "cell-centered" framework; i.e. their numerical values correspond to the evaluation of the fields at the center of the grid cells. Each field must be attached to a topology object, which describes the size and the memory arrangement of the field, as will be detailed in Section 3. The initialization of FLUPS always starts with the precomputation of the communication patterns and with the planning of the FFTs, which depend on the user-specified BCs and on the topology of the RHS field, $f$. The evaluation of the user-specified Green's function is also performed during the setup of the library, and the Green's function in the full spectral space $\hat{G}$ is eventually obtained and stored. Then, for any given RHS, the solution is obtained by first computing $\hat{f}$, i.e. the forward 3D FFT of $f$, by multiplying it with $\hat{G}$, and by computing the backward transform of the solution. Each of the following sections focuses on the specific stages of this process.

2.1. Evaluation of Green's function for spectral and unbounded directions. Following the numbering of the introduction, the Green's functions available in FLUPS are (I) the singular expressions with replacement of the singularities by cell-average values as in [4]; (II) those of [18, 30] with explicit regularization up to the order $m=10$ and their spectral generalization presented in [19] ; and (IV) the lattice Green's functions of [24]. The analytical expressions of the Green's functions in cases (I) , (II) and (IV) are summarized in Appendix B. Depending on the BCs, additional processing (including FFTs) is required to obtain $\hat{G}$.

2.1.1. Fully unbounded problems. For fully unbounded problems the Green's function is expressed in the physical space. Indeed, the singular Green's functions of type (I) are obtained as the solution of

$$
\nabla^{2} G=\delta(\mathbf{x}),
$$

and the regularized type (II) Green's functions are obtained from

$$
\nabla^{2} G_{m}=\zeta_{\varepsilon, m}(\mathbf{x})
$$

where the analytical expression of $G$ is known as a function of the spatial coordinates. The domain doubling technique must be used to obtain the result in the Fourier space (thus involving a 3D FFT); see Section 2.2.2. In (2.2), $\zeta_{\varepsilon, m}$ is a regularization kernel, as in [18]. It is here assimilated to a Gaussian mollification of order $m$ and of characteristic smoothing length $\varepsilon=2 h$ (see Appendix A for their mathematical expressions).

2.1.2. Fully spectral problems. Conversely, if all directions are spectral (i.e., with periodic or symmetric BCs), the Green's function is readily expressed in the full-spectral space. For instance, (2.2) is expressed in the Fourier space as (ik) $\cdot(\mathbf{i k}) \hat{G}=\hat{\zeta}_{\varepsilon, m}(\mathbf{k})$, which yields for $\hat{G}$

$$
\hat{G}_{m}=-\frac{1}{\mathbf{k} \cdot \mathbf{k}} \hat{\zeta}_{\varepsilon, m}(\mathbf{k})
$$

where $\mathbf{k}=\left[k_{x}, k_{y}, k_{z}\right]$, with $k_{i} \in\left[-\pi / h_{i} ; \pi / h_{i}\right]$. No FFTs are hence required to evaluate the Green's function.

2.1.3. Partially unbounded problems. For domains combining one (resp., two) spectral direction and two (resp., one) infinite (or semi-infinite) directions, Green's functions are obtained from the solution of a Helmholtz equation. Indeed, the Laplacian operator is split between the unbounded and the periodic/symmetric direction, and one considers the partial Fourier transform of (1.1) along the latter direction, which hence becomes spectral [4]. This results in a Helmholtz equation, which is expressed as a function of the wave number $k$ in the spectral direction and as a function of the spatial coordinates in the unbounded directions. For example, considering two unbounded directions $X, Y$ and a periodic direction $Z$, the regularized Green's function is obtained as the solution of

$$
\nabla_{x, y}^{2} \tilde{G}\left(x, y, k_{z}\right)-k_{z}^{2} \tilde{G}\left(x, y, k_{z}\right)=\hat{\zeta}_{\varepsilon, m}\left(k_{z}\right),
$$

where . denotes the partially transformed function. The remaining spatial directions are then to be transformed using FFTs and the domain doubling technique. 
2.2. 3D FFT transform and convolution. Numerically, the $3 D$ FFT is obtained as the succession of $1 D$ FFTs in all three directions, and each transform type is driven by the BCs in the considered direction. For example, starting from $f(x, y, z)$ in spatial coordinates and seeking for the fully periodic transform, one thus obtains successively $\tilde{f}\left(x, y, k_{z}\right)$ as the result of the first DFT in $z, \tilde{f}\left(k_{x}, y, k_{z}\right)$ as the result of the second DFT in $x$, and finally $\hat{f}\left(k_{x}, k_{y}, k_{z}\right)$ as the result in all-spectral coordinates. The type of $\mathrm{BC}$ dictates the order of the transforms, and hence the order of treatment of the directions, so as to minimize the number of computational operations (see Section 3).

In a fashion much akin to the P3DFFT library, FLUPS uses pencil decompositions of the domain. From one FFT operation to another, a change in topology is thus required-here called topology switch. The latter involves a very large number of communications, and a significant effort was dedicated to reducing the associated latency in FLUPS (see Section 3). Additionally, since all 1D FFT operations (here conducted using the FFTW library [10]) require the contiguity of data in memory along the transformed direction, a memory transpose is also necessary to reorder the data along each pencil direction. Notice that non-unit stride 1D FFTs are also implemented in FFTW, but their use would here lead to a slower execution.

In the following subsections, the treatment of the transform in each direction is described, with a focus on the particularities of the various types of BCs. Moreover, the operations on the field $f$ and on the field $G$ are detailed when appropriate. The final result of the 3D FFT hence consists in a succession of these treatments for all the directions, which are processed separately as 1D problems.

2.2.1. Spectral direction. The spectral (symmetric or periodic) directions are the most straightforward. With periodic BCs, the forward Fourier transform of $f$ is readily obtained using a Direct Fourier Transform (DFT). For symmetric directions, and accounting for the fact that fields $f$ and $\phi$ are "cell-centered", symmetry conditions are implicitly satisfied at the cell edges using

- the type-II DST for odd-odd cases,

- the type-IV DST for odd-even cases,

- the type- $I V$ DCT for even-odd cases,

- the type- $I I$ DCT for even-even cases.

The latter are equivalent to, yet much more efficient than, the use a DFT on a domain of size $2 N$ (for odd-odd and even-even cases) or $4 N$ (for crossed cases), where the symmetries would be explicitly enforced by a copy of the data into a symmetric image in the extended domain. We refer the reader to [10] and to the documentation of the FFTW package ${ }^{1}$ for complete definitions. Notice that the backward transform of $\hat{\phi}$ is performed with the corresponding DFT/DCT/DST type, as the solution field enjoys the same symmetries as the RHS.

THe Green's function needs not undergo an FFT in the spectral directions as the analytical expression is already expressed as a function of the spectral coordinates for both periodic and symmetric BCs. However, the wave number $k$ used for its evaluation (i.e. the wave number of the current direction) must correspond to the modes resulting from the transform of the source field. Considering the definition of the various types of transforms,

- the type- $I I$ DCT is given by

$$
\tilde{f}=2 \sum_{j=0}^{N-1} f\left(x_{j}\right) \cos \left[\pi\left(j+\frac{1}{2}\right) \frac{\mathrm{k}}{N}\right]
$$

where the kth entry in the result of the DCT corresponds to the mode $k$. The flip-flop mode, $\mathrm{k}=N$, is trivially null and the Green's function $\tilde{G}$ is thus evaluated between $k=0$ and $k=N-1$;

- the type- $I I$ DST is given by

$$
\tilde{f}=2 \sum_{j=0}^{N-1} f\left(x_{j}\right) \sin \left[\pi\left(j+\frac{1}{2}\right) \frac{\mathrm{k}+1}{N}\right]
$$

where the kth entry in the result of the DST corresponds to the mode $k+1$ since the mode $k=0$ is trivially null. $\tilde{G}$ is thus evaluated between $k=1$ and $k=N$;

- the type- $I V$ DCT corresponds to

$$
\tilde{f}=2 \sum_{j=0}^{N-1} f\left(x_{j}\right) \cos \left[\left(\mathrm{k}+\frac{1}{2}\right) \pi \frac{\left(j+\frac{1}{2}\right)}{N}\right],
$$

and the first entry $\tilde{f}(\mathrm{k}=0)$ is actually the global mode $k=1 / 2$. $\tilde{G}$ is thus correspondingly evaluated with an offset of $\frac{1}{2}$. The same conclusion holds for the type- IV DST.

Notice that the forward transform of $f$ in periodic directions uses a real to complex or a complex to complex transform, depending on the input data type. The backward transform of $\hat{\phi}$ makes use of the corresponding inverse operation.

\footnotetext{
${ }^{1}$ The documentation of the FFTW library is available online at www.fftw.org/fftw3_doc.
} 
2.2.2. Fully unbounded direction. Following the domain doubling technique by Hockney and Eastwood [20], $f$ is extended to a domain of size $2 N$ with zero-padding; the Green's function is extended to $2 N$ and symmetrized about the Nth data, as in the example shown in Figure 1a and Figure 1b. The unbounded (i.e. aperiodic) convolution is obtained through the convolution in the doubled domain with periodic BCs. Figure 1c, Figure 1d and Figure 1e illustrate the operations involved in that convolution, as if it was performed in spatial coordinates, with periodicity over the doubled domain of size $2 L$. One easily verifies that performing the convolution between the padded fields with periodic BCs yields the same results as if the domain was unbounded in the nonpadded region $(x \in[0, L])$. The result in the padded region $(x \in] L, 2 L])$ is spurious and is hence discarded. One thus obtains $\tilde{f}$

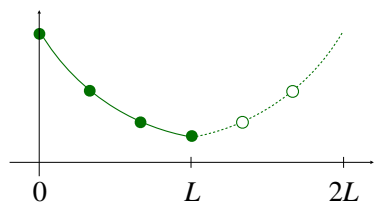

(a) Green's function $G$

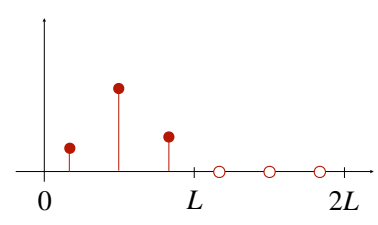

(b) right hand side $f$

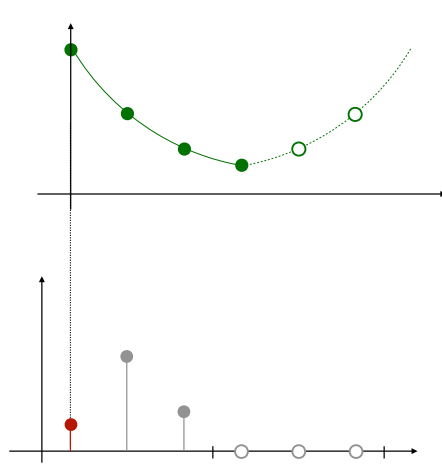

(c) convolution term $j=0$

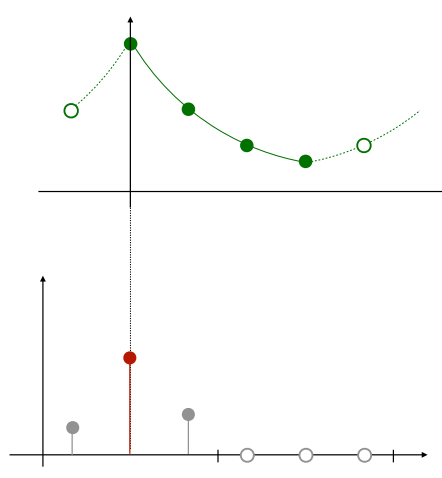

(d) convolution term $j=1$

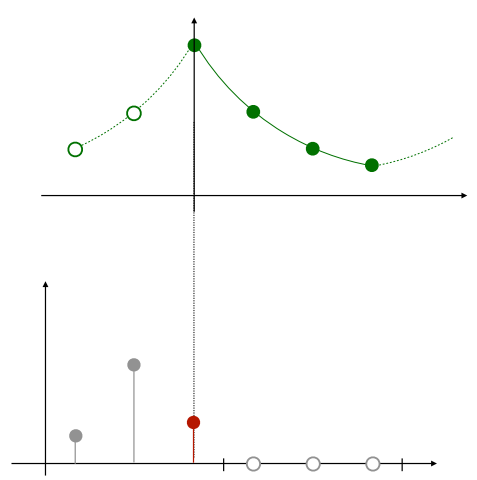

(e) convolution term $j=2$

Figure 1: Example of the steps involved in a convolution between the Green's function and a source term, $\phi\left(x_{i}\right)=\sum_{j=0}^{N-1} G\left(x_{j}-\right.$ $\left.x_{i}\right) f\left(x_{j}\right)$. The filled bullets show the data in the initial domain $x=[0, L]$ of size $N=3$. The empty bullets denote the data in the extension of the domain, with symmetry on $G$ about $x=L$ and zero-padding on $f$.

and $\tilde{G}$ as the the forward transform of $f$ and $G$ using DFTs over the doubled domain, again of type real to complex or complex to complex, depending on the input data type.

As previously mentioned, the convolution in FLUPS is implemented through the multiplication in Fourier space. It is to be noted that, because of the domain extension and the implicit periodicity, the Green's function and the field eventually have the same size of $2 \mathrm{~N}$ unknowns in the Fourier space. Hence, no special care needs to be taken for the computation of the point-to-point multiplication.

2.2.3. Semi-unbounded direction. A semi-unbounded direction is a direction with a symmetry condition at one end and an infinite BC at the other end. Conceptually, this configuration is easily modeled with an extension of the domain. As illustrated in Figure 3 , the source term is first copied so as to explicitly satisfy the BC, hence extending the computational domain from $[0, L]$ to $[-L, L]$ in the case of a symmetry condition on the left side of the domain (or to $[0,2 L]$ in the case of a symmetry condition on the right side of the domain). The resulting configuration is unbounded on a domain of size $2 L$ and can be solved as described in the previous section: the Green's function is evaluated over $[0,2 L]$ and symmetrized in the padded region, [2L, $4 L]$, and a DFT is used to obtain the data in the spectral space. However, strictly applying this would require a domain of size $4 N$ (because of two successive domain doublings), which is not acceptable for performance reasons.

Instead, DSTs and DCTs are here exploited to reduce the computational cost, while still imposing the correct symmetry condition at the desired domain end. In this approach, the domain doubling is still performed as for the fully unbounded direction, but the zero-padding is here done on the side which is unbounded. In the example considered in Figure $2 \mathrm{~b}$, it takes place on the right side, $] L, 2 L]$. The domain over which the DST/DCT will be executed is hence $[0,2 L]$. As for the Green's function (Figure $2 a$ ), it must be here evaluated in the extended domain and transformed using a DCT (whereas it was explicitly symmetrized in the extended domain for fully unbounded domains; see Figure 1a). As shown in Figure 3, this guarantees that the convolution accounts for the proper BCs on the domain $[0,2 L]$.

As shown in Figure 2a, the Green's function is evaluated in a "vertex-centered" framework, as it characterizes the influence of a source located in $\mathbf{x}=\mathbf{0}$ on any other location in the domain. Most importantly, $f$ is in a cell-centered layout. Therefore different types of Fourier transforms have to be used to compute $\tilde{f}$ and $\tilde{G}$ with the proper symmetry conditions. In order to guarantee that their product is consistent with the aimed convolution, the kth entry in the result of the FFT of $f$ must be related to the corresponding mode $k$ of $\tilde{G}$.

Practically, the field $f$ (with $2 N$ cell-centered real data, as in Figure 2 b) will be transformed into $\tilde{f}$ using a type- $I I$ DCT for an 


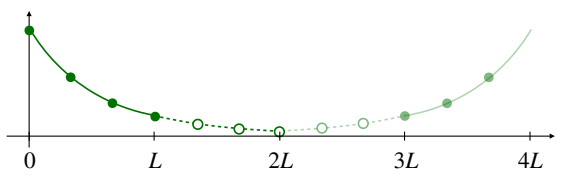

(a) Green's function $G$ extended for a semiunbounded direction

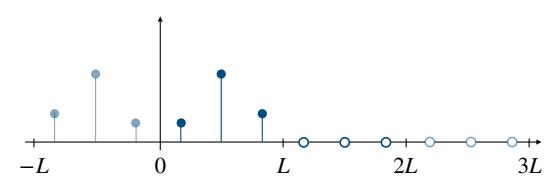

(b) right hand side $f$ with even symmetry on the left

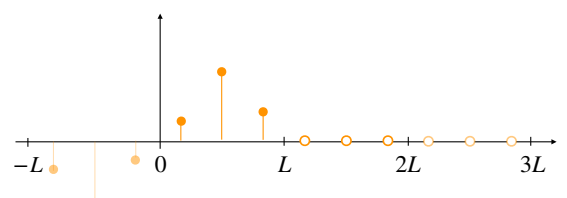

(c) right hand side $f$ with odd symmetry on the left

Figure 2: Green's function and RHS initially on a domain $x=[0, L]$ of size $N=3$, and their virtual extension in a domain of size $4 L$ for the resolution of a semi-infinite direction with symmetry on the left. Faded colors indicate ghost data that are not present when using a DCT or a DST to enforce the symmetry. Only the portion $[0,2 L]$ is then effectively evaluated.

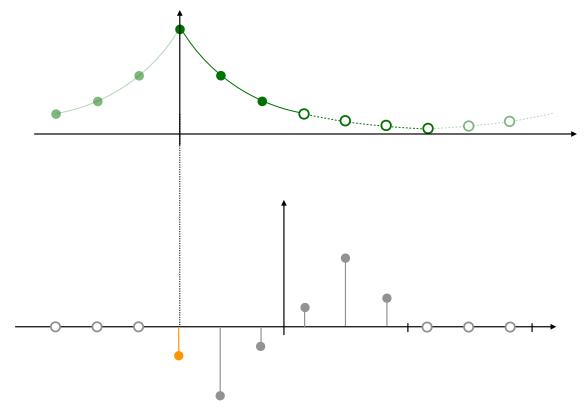

(a) convolution for $j=4$

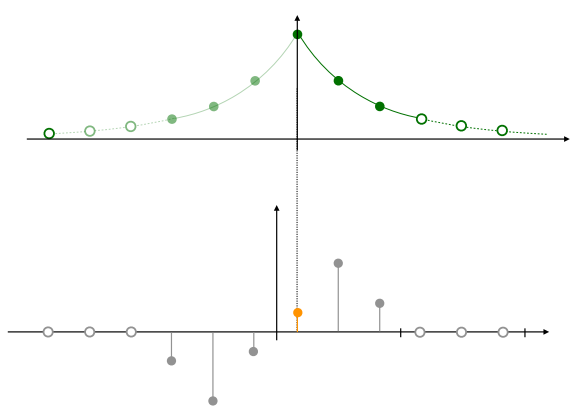

(b) convolution for $j=7$

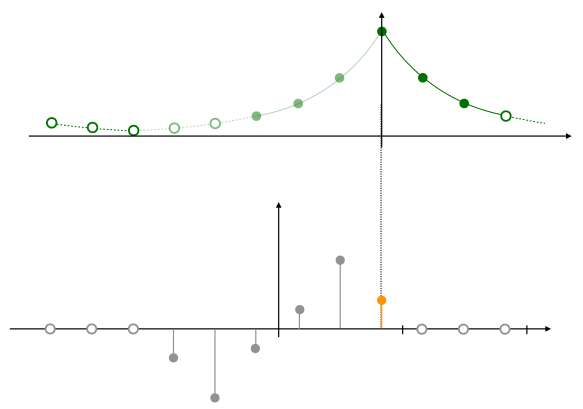

(c) convolution for $j=9$

Figure 3: The convolution of $G$ (top) with $f$ (bottom) on a $4 N$ size domain padded for a semi-unbounded transform with odd symmetry on the left. The correct solution is retrieved in $x=[-L, L]$.

even symmetry, and using a type- $I I$ DST for an odd symmetry, which both return $2 N$ numbers (real or complex; the same kind as $f$ ). According to their definitions ((2.5) for the DCT; (2.6) for the DST) when expressed in the case of a domain with $2 N$ cells, the flip-flop mode $(k=2 N)$ is trivially zero in the type- $I I$ DCT, while the mode $k=0$ is trivially zero in the type- $I I$ DST. On the other hand, the $2 N+1$ numerical values of the Green's function (evaluated on the vertices of the interval [0,2L], as in Figure $2 \mathrm{a}$ ) are transformed using a type- $I$ DCT, which yields $2 N+1$ values:

$$
\tilde{G}=G\left(x_{0}\right)+(-1)^{\mathrm{k}} G\left(x_{2 N}\right)+2 \sum_{j=1}^{2 N-1} G\left(x_{j}\right) \cos \left[\pi j\left(\frac{\mathrm{k}}{2 N}\right)\right] \text {, }
$$

where the kth entry corresponds to mode $k$ and neither the constant mode $k=0$ nor the flip-flop mode $k=2 N$ is null. Hence particular care must be taken to match the entries of $\hat{f}$ and $\hat{G}$ with the same mode $k$ while performing the pointwise multiplication in Fourier space. By doing so, one finally obtains the field $\hat{\phi}$ of size $2 N$, with the first or the last mode being trivially null and ready for the application of the backward transform.

2.2.4. Order of the transforms. The order in which the directions are transformed to obtain the 3D FFT can be chosen arbitrarily. As the DCTs and DSTs are real to real transforms (for symmetric and semi-unbounded directions), one may want to avoid performing them on complex data, i.e. separately on the real and on the complex parts. Therefore, the symmetric directions are always treated first, in order to reduce the size and the cost of subsequent FFT evaluations (which can thus be real to real, or real to complex). Then, the semi-unbounded directions are transformed, which yields real values on a doubled domain. Finally, the FFTs in the periodic directions and in the unbounded directions are performed, using real to complex or complex to complex transforms, depending on the input data type.

FLUPS follows these directives to determine the actual order of executions of the FFTs. This order is stored in the dimorder array.

\section{Additional notes on the parallel implementation.}

3.1. Topologies and data layout. As already mentioned, FLUPS uses topology objects in order to describe every field in the memory. The topology specifies the useful information on the field, including the global and local numbers of field data in each 
direction, the type of data $\left(\mathrm{n}_{\mathrm{f}}=1\right.$ for real data and $\mathrm{n}_{\mathrm{f}}=2$ for complex data), the number of MPI ranks in each direction $N_{c}^{x}, N_{c}^{y}, N_{c}^{z}$, and the associated MPI communicator. If the communicator is of type MPI_Cart, the information on the mapping between the cores and the physical domain is retrieved from the communicator itself, otherwise it needs to be specified by the user.

Most importantly, every topology also has its own specific fastest rotating index (FRI), axo, i.e. an integer indicating the axis ( $X=0, Y=1$ or $Z=2$ ) along which data are contiguous in memory. The 3D data are stored sequentially in memory: data are decomposed in the order $\mathrm{ax}_{0}, \mathrm{ax}_{1}, \mathrm{ax}_{2}$, where the last two indices follow a natural permutation of indices, $\mathrm{ax}_{1}=\left(\mathrm{ax}_{0}+1\right) \% 3$ and $\mathrm{ax}_{2}=\left(\mathrm{ax}_{0}+2\right) \% 3$. As an example, for a 3D data set of size $N=\left[N_{x}, N_{y}, N_{z}\right]$ with an FRI aligned with $\mathrm{ax}_{0}=1$ (and thus with $\mathrm{ax}_{1}=2$ and $\left.\operatorname{ax}_{2}=0\right)$, the element located in $(x=10, y=2, z=3)$ will be located in memory at $\left[2\left(\mathrm{n}_{\mathrm{f}}\right)+3\left(\mathrm{~N}_{\mathrm{y}} * \mathrm{n}_{\mathrm{f}}\right)+10\left(\mathrm{~N}_{\mathrm{z}} \mathrm{N}_{\mathrm{y}} * \mathrm{n}_{\mathrm{f}}\right)\right]$.

The size of the computational domain may be extended along the FRI in order to fulfil data alignment requirements. Indeed, only one FFTW plan is defined per pencil topology, and it is used to transform all the pencils in the domain. For this to be possible, the starting element of each pencil must be aligned in memory. Therefore, if the size of one pencil does not match a multiple of the memory chunk size, the domain is padded in the FRI dimension to ensure the correct alignment of all pencils.

In the other dimensions, the sizes of the pencils are chosen in order to ensure load balancing: the width of all pencils in a given direction differs by 1 at most. For example, if the $x$ direction with $N_{x}=20$ was to be split between three cores, each core would be in charge of, respectively, 7, 7, and 6 data.

3.2. Memory management and computational complexity. In order to avoid repetitive large allocations of memory, the whole solver is designed to work "in place". The total amount of memory required for the solve operation is allocated once at initialization. The size of the allocation, which corresponds to the maximum size that the data will occupy, is determined by the execution of a dry run. This procedure accounts for the directions that will need zero-padding, and also predicts the type of FFT transform (real to real, real to complex, complex to complex) in order to determine the input and output sizes. This information is used to create three topologies with pencils in each direction that will enable the series of 1D FFTs, to determine the optimal order dimorder in which these topologies will be used, and to prepare the switches between the successive topologies.

Even though the size of the memory allocation corresponds to the maximum size of the problem (accounting for domain doubling), FFTs are only performed on the significant part, i.e., not on zero-padded data. For instance, a 3D unbounded problem of size $N \times N \times N$ requires a total memory allocation of $(2 N+2) \times(2 N+2) \times(2 N+2)$ floating point values, but the computational complexity associated with the forward (or backward) transform ${ }^{2}$ is as small as $7 N^{2} \mathscr{O}(N \log (N))$. If the FFTs were all heedlessly computed on the full size domain $(2 N+2)^{3}$, the complexity would be $12 N^{2} \mathscr{O}(N \log (N))$-almost twice as large. This can also be compared to the cost of a fully periodic transform without padding, $3 N^{2} \mathscr{O}(N \log (N))$.

As another example, a domain with odd-odd symmetry conditions in the first two dimensions, and fully unbounded in the third direction, leads to a memory allocation of $N \times N \times(2 N+2)$ floating point values. First, $f$ is transformed twice in the symmetric directions using $N \times N$ DSTs of size $N$ (hence excluding the zero-padded region). Then, the fully unbounded direction is transformed using $N$ DFTs with $2 N$ real numbers as an input and with $N+1$ complex numbers as an output (thus corresponding to $2 N+2$ values). By explicitly discarding the zero-padded region for the first two DSTs, the total cost of one transform is $4 N^{2} \mathscr{O}(N \log (N))$.

3.3. Creation of topologies and topology switches. Again, during the initialization of FLUPS, three pencil topologies dedicated to each of the 1D FFTs are first created. These topologies have an FRI aligned with the direction of the pencils. For an illustration of the pencil decompositions, the reader is referred to Figure 2 in [6], which follows the same idea. Three switches are also set up. The first one is dedicated to remapping data from the physical topology to the first pencil topology, and the two others work as pencil-to-pencil.

When creating the new pencil topologies, FLUPS is free to choose the decomposition regardless of the physical topology. In particular, the number of pencils in each direction can be optimized based on the predetermined order of the switch operations (as described in Section 2.2.4). This number is here chosen in order to take advantage of the interfaces between MPI ranks that are common in the origin and the destination topologies. Indeed, the common interfaces partition the domain into subsets of ranks: if a rank belongs to a subset in the input topology, it belongs to the same subset in the output topology. Moreover, each of the subsets contains a smaller number of ranks than the total $N_{c}^{x} N_{c}^{y} N_{c}^{z}$. Inside the subset, they are to communicate in an all-to-all fashion during the execution of the topology switch. One sub-communicator can thus be created per subset, and the reduced size helps in lowering the communication latency on very large partitions. For this to work, one further needs to ensure that rank numbering is consistent in both input and output topology communicators.

Consider a physical topology with the number of ranks per direction $\left[\mathrm{N}_{\mathrm{C}}^{\mathrm{X}}=2, \mathrm{~N}_{\mathrm{c}}^{\mathrm{Y}}=2, \mathrm{~N}_{\mathrm{c}}^{\mathrm{z}}=2\right]$, and a dimorder $=[1,0,2]$, as illustrated in Figure 4a. First, the rank IDs are split following the 3D decomposition given by dimorder, which yields a 3D index. For example, the rank 2 will have the $3 \mathrm{D}$ index $[1,0,0]$, since $0+1 * 2+0 * 4=2$. This is to ensure that a rank which is located in one sub-communicator will remain in the same sub-communicator in the next topology. Then, FLUPS sets up the pencil decomposition: the sizes of the first two directions in the dimorder array are multiplied, while the last one is left unchanged. In this case, $\left[\mathrm{N}_{\mathrm{c}}^{\mathrm{x}}=4, \mathrm{~N}_{\mathrm{c}}^{\mathrm{y}}=1, \mathrm{~N}_{\mathrm{c}}^{\mathrm{Z}}=2\right]$, hence creating 4 sub-communicators because of the 2 common interfaces. Afterwards, FLUPS permutes the number of ranks to reach the second pencil decomposition, $\left[\mathrm{N}_{\mathrm{C}}^{\mathrm{X}}=1, \mathrm{~N}_{\mathrm{C}}^{\mathrm{Y}}=4, \mathrm{~N}_{\mathrm{C}}^{\mathrm{Z}}=2\right]$, creating 2 sub-communicators, as depicted in Figure $4 \mathrm{~b}$. Finally FLUPS would permute the last two directions of the dimorder array to obtain $\left[\mathrm{N}_{\mathrm{c}}^{\mathrm{X}}=2, \mathrm{~N}_{\mathrm{c}}^{\mathrm{y}}=4, \mathrm{~N}_{\mathrm{c}}^{\mathrm{z}}=1\right]$, creating

\footnotetext{
${ }^{2}$ The leading complexity of an FFT over a doubled domain is $2 N \log (2 N)=2 N(\log (2)+\log (N))$ thus still $\mathscr{O}(N \log (N))$.
} 
4 sub-communicators (not presented in the figure). This strategy ensures that the last topology switch, which should involve the largest volume of transferred data due to the padding for unbounded directions, uses as many sub-communicators as possible (and thus decoupled communication patterns).

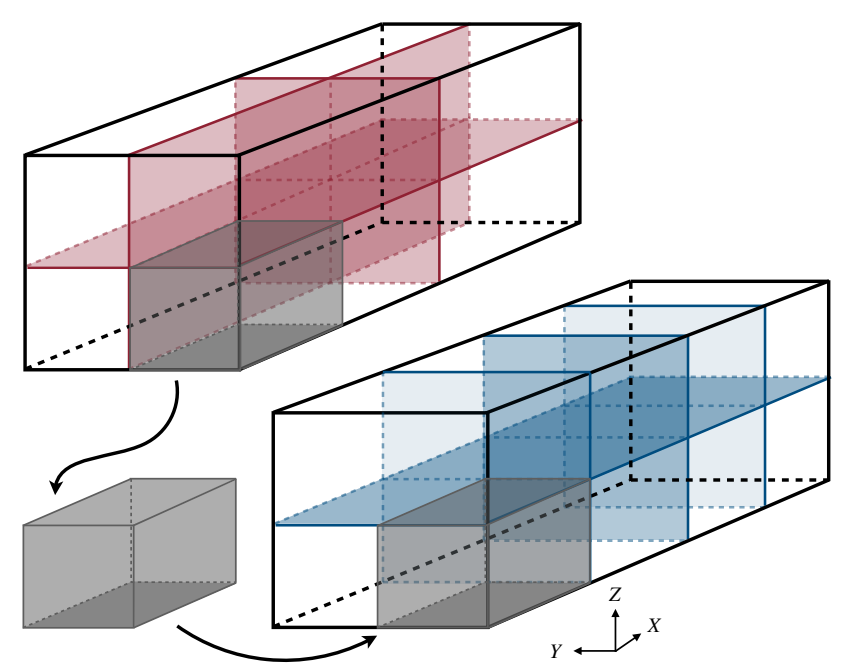

(a) Switch from the physical topology (in red) to the first pencil topology aligned in the $Y$ direction (in blue). The grey area represents interface between ranks, highlighted for interfaces shared by both topologies.

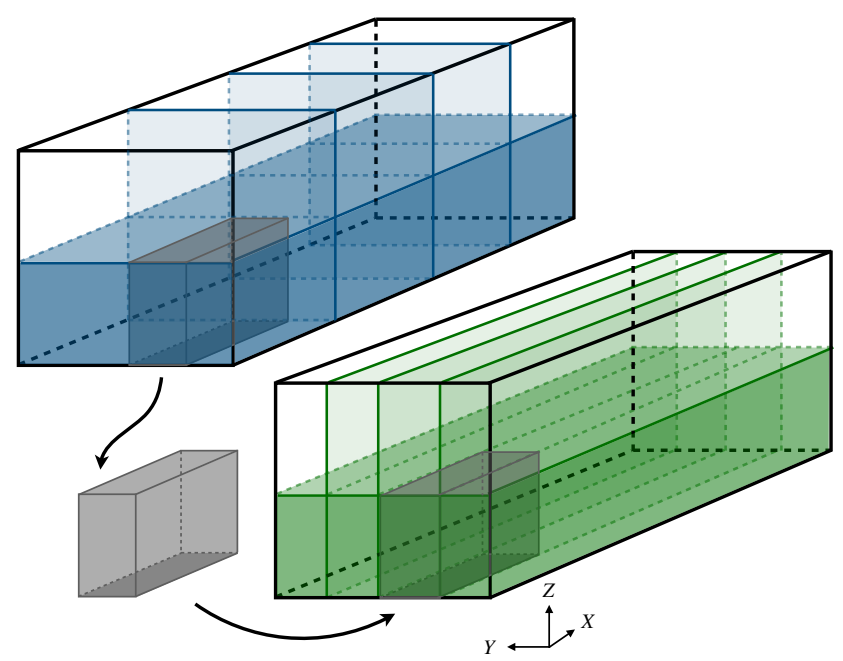

(b) Switch form the first pencil topology aligned in the $Y$ direction (in blue) to the second pencil topology aligned in the $X$ direction (in green). One of the two sub-communicators associated to this topology switch is highlighted in the corresponding topology's color.

Figure 4: Illustration of the topology switches that FLUPS would define starting from a physical topology with 8 MPI ranks, 2 in each direction (in red). Examples of communication blocks are shown in grey.

MPI is used to communicate between the cores when switching from one topology to another. Prior to the communication, data in memory are grouped in blocks, defined as the largest possible subset of the domain that will be either sent to or received from a given rank (as shown in grey in Figure 4). During the initialization of FLUPS, the size of those blocks and the communication patterns (origin and destination of each blocks) are determined and stored. The maximum total size of the communication buffers is also precomputed so that only one memory allocation is performed, and they are reused for the three topology switches, akin to what is done for the field data.

The execution of a topology switch starts with the copy of the blocks into the send buffers. As the blocks are made of several chunks of contiguous memory, this operation benefits from autovectorization along the FRI. The start address of each chunk is not necessarily aligned in memory, but when possible, instructions for aligned memory access are issued. When the send buffers are filled, one proceeds to the communication. More details on this operation are provided hereafter. As a result, new data are available in receive buffers. Since the FRI changes between the origin and the destination topologies, these data need to be transposed in memory to realign them in the correct direction. To do so, one option would consist in looping over the indices of the destination topology and simply copying back values from the buffer to the field. This, however, would result in slow, non-unit stride memory accesses. Instead, one here rather resorts to the FFTW library to efficiently "shuffle" the data in the buffer, through the functions $f f t w \_p l a n_{-} g u r u_{-} r 2 r$ for real data and $f f t w \_p l a n_{-} g u r u_{-} d f t$ for complex data. The data can then be copied from the buffers back into the field, again benefiting from autovectorization and, potentially, aligned access.

3.4. Communication strategies. For the communication in itself, two different methods are made available to the user, as the optimal communication scheme will depend on the implementation of the MPI standard as well as on the targeted hardware. The first communication method is implemented using calls to the MPI_alltoall and MPI_alltoallv routines. When all blocks across a whole sub-communicator have the same size, FLUPS automatically uses the MPI_alltoall routine, as it is believed to sometimes outperform its variable-size counterpart thanks to more advanced internal optimizations. In all other cases, the MPI_alltoallv routine is used.

In the second method, all communications are handled using MPI non-blocking persistent send and receive calls, detailing explicitly the origin, the destination and the size of each block. The communication patterns are precomputed once and stored at initialization, hence sparing some time during the execution of the switches. Even though point-to-point send and receive operations are generally expected to be slower than the all-to-all communication (which benefits from global network optimization), a slight performance gain is here expected from the non-blocking aspect. Indeed, as soon as a block is received by a core (i.e., when the MPI_waitany routine returns), the copy from the corresponding receive buffer to the main memory can start, while the process is still waiting to receive the other blocks. In practice, as soon as the communication is initiated, each process starts by 
copying the block that remains on that rank (if there is one).

FLUPS is able to exploit shared-memory parallelism, when the user requires threads. If so, all the copy operations (from/to buffers for instance) are accelerated with OpenMP parallel for instructions. In addition, OpenMP is also used to parallelize the FFTs. The total number of 1D transforms to be performed in a pencil topology is split between the available threads.

As FLUPS supports hybrid parallelism, for a given number of cores, an optimum is likely to be found between the number of threads and the number of MPI ranks. Generally, for a decreasing number of MPI ranks (i.e. an increasing number of threads), the size of the blocks increases and one expects the communication latency to decrease, up to a point where the transfer speed does not depend on the size of the message. The communication latency mainly depends on the network architecture of the targeted computer, and on the network congestion (see Section 5 for timings).

3.5. Rank reordering. An additional optimization of FLUPS was designed to potentially adapt the distribution of the MPI rank indices to the actual network architecture. As the communication pattern between the ranks is known a-priori for each topology switch, one may want to optimize globally the position of each rank in the hardware communication network, in order to reduce the congestion of the network during the communications, and to avoid any bottleneck. Such an optimization reduces to permuting the rank indices between cores in the physical topology adequately, resulting in a given rank (associated to a given portion of the 3D domain) located at the desired location in the network. The determination of the optimal permutation-which should account for a specified communication graph, for the actual network architecture and for the resources allocated-is beyond the scope of this paper. However, according to the MPI specifications, this task is supposedly achieved by the MPI_Dist_graph_create_adjacent routine when the reordering of the ranks is requested. To exploit it, the communication graph between the MPI ranks is established at the initialization of FLUPS: an edge exists between ranks $i$ and $j$ if they need to communicate during one of the pencil to pencil topology switches, and the associated edge weight is computed as the total amount of data that will be exchanged during the forward and backward transforms. Based on the weighed graph, MPI_Dist_graph returns a new communicator where the rank indices are "smartly" permuted. FLUPS then recomputes the communication pattern of the first topology switch in order to ensure that the data in the user topology will be routed to the correct core with the new numbering in the first pencil topology. As a result, a bit more data needs to be exchanged during the execution of the first topology switch, between the user-specified physical topology with the original communicator and the first pencil topology with the new communicator. The potential increase in communication delay related to this operation should be balanced by the gain over the subsequent topology switches. Optionally, the optimized communicator can be retrieved by the user so that it can be used from the beginning of the computation on the user side, thus avoiding the data rerouting of the first topology switch.

FLUPS is thus ready to exploit such an optimization. In practice, however, it was not possible to demonstrate the efficiency of this feature. At the time of this writing, the authors could not find an MPI implementation with documentation on the reordering. Several tests were executed on different machines using Intel's implementation of MPI (see Section 5), but none of them led to rank indices actually permuted at the output of MPI_Dist_graph. The result presented in the following sections are hence obtained with the canonical rank ordering.

4. Validation. In this section, an extensive validation of FLUPS is presented for the Poisson equation (reproduced from (1.1))

$$
\nabla^{2} \phi=f
$$

with various combinations of BCs, in 3D. The convergence of the solver is measured using the infinite norm of the error (as the infinite norm bounds the 2-norm), defined as

$$
E_{\infty}=\sup _{x, y, z}\left\{\left|\phi(x, y, z)-\phi_{r e f}(x, y, z)\right|\right\}
$$

where $\phi_{\text {ref }}$ is an analytical solution. For the latter, a composite expression is employed:

$$
\phi_{\text {ref }}(x, y, z)=\phi_{x}(x) \phi_{y}(y) \phi_{z}(z)
$$

The 1D functions $\phi_{x}, \phi_{y}$, and $\phi_{z}$ are chosen to satisfy the set of BCs. Simple analytic expressions are preferred so that meaningful numerical solutions can be obtained even with small resolutions, hence allowing us to verify the slopes of the convergence more easily. For instance, sine and cosine are used in the periodic and symmetric directions, with an appropriate wavelength. Fully unbounded solutions are guaranteed with a compact Gaussian function, i.e., $\phi_{x}(x)=\exp \left(\mathscr{C}\left(1-\frac{1}{\left(1-\left(\frac{x-x_{c}}{\sigma}\right)^{2}\right)}\right)\right)$ for $-1<\frac{x-x_{c}}{\sigma}<$ 1 , and $\phi_{x}=0$ elsewhere. The dimensional parameter $\sigma$ and the center of the blob $x_{c}$ are set so that the value of the RHS at the unbounded boundaries is null. The constant $\mathscr{C}$ is arbitrarily set to 10 . For semi-infinite directions, a linear combination of compact Gaussian functions is used. The parameter $x_{c}$ and the sign of each term are chosen in order to explicitly impose the symmetric BC. Given these analytical expressions, the RHS $f$ is computed as

$$
f(x, y, z)=\frac{d^{2} \phi_{x}}{d x^{2}}(x) \phi_{y}(y) \phi_{z}(z)+\phi_{x}(x) \frac{d^{2} \phi_{y}}{d y^{2}}(y) \phi_{z}(z)+\phi_{x}(x) \phi_{y}(y) \frac{d^{2} \phi_{z}}{d z^{2}}(z)
$$


In FLUPS, the BCs in a single direction can be set to periodic, or to a pair of options among even symmetry, odd symmetry, and unbounded. This leads to a thousand possible combinations of BCs in 3D. For the sake of concision, only three cases of validation are presented here. All the other configurations were tested and validated as part of an automated process during the development of the library. For the interested reader, FLUPS provides a C++ sample code dedicated to the test and the validation of the library. When built, the associated executable can be easily used to reproduce the convergence results.

4.1. Domain with symmetric and periodic BCs. The Poisson equation is here solved on a cubic domain of spatial extent $[0, L]$ in all directions, with even-even symmetry conditions in $X$, odd-even symmetry conditions in $Y$, and periodic in $Z$. The chosen reference solution is

$$
\phi_{\text {ref }}(x, y, z)=\cos \left(2 \pi \frac{x}{L}\right) \cos \left(\frac{9 \pi}{2} \frac{y}{L}\right) \sin \left(8 \pi \frac{z}{L}\right) .
$$

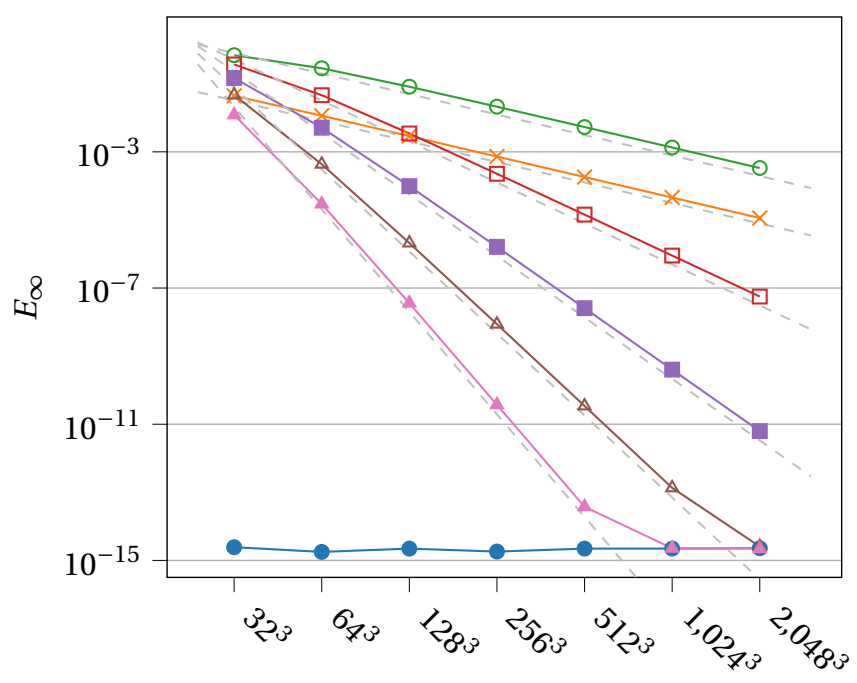

$\mathrm{N}$

Figure 5: Convergence of FLUPS with symmetric and periodic BCs: - CHAT2 and HEJ0 spectral, $*$ LGF2 $\mathscr{O}\left(h^{2}\right),-\circ$ HEJ2 $\mathscr{O}\left(h^{2}\right), \square \operatorname{HEJ} 4 \mathscr{O}\left(h^{4}\right),-\operatorname{HEJ} 6 \mathscr{O}\left(h^{6}\right), \triangle \operatorname{HEJ} 8 \mathscr{O}\left(h^{8}\right),-\operatorname{HEJ} 10 \mathscr{O}\left(h^{10}\right)$.

The error obtained with FLUPS is measured in terms of infinite norm for all the implemented Green's functions: the singular expression (CHAT2, which is in the present case equivalent to HEJO), the regular expressions with order up to $m=10$ (HEJ2,HEJ4,HEJ6, HEJ8 and HEJ10), and the lattice Green's function LGF2. Their mathematical expressions can be found in Appendix B.4. The results are shown in Figure 5, as a function of the resolution per direction of the domain. Convergence is indeed observed, at the order corresponding to the theoretical order of each method. As the present test case is fully spectral, the singular Green's function used by CHAT2 provides the exact (spectral) solution, and the error thus amounts to the machine precision regardless of the resolution.

4.2. Domain with three fully unbounded BCs. Unbounded BCs are now used in all directions, on the same domain. The reference solution is

$$
\phi_{r e f}(x, y, z)=\exp \left(10\left(3-\frac{1}{\left(1-\left(\frac{2 x}{L}-1\right)^{2}\right)}-\frac{1}{\left(1-\left(\frac{2 y}{L}-1\right)^{2}\right)}-\frac{1}{\left(1-\left(\frac{2 z}{L}-1\right)^{2}\right)}\right)\right) .
$$

The convergence results obtained with the Green's functions (recalled in Appendix B.1) are shown in Figure 6. Again, we retrieve the expected convergence behavior. Moreover, the additional spectrally truncated HEJO kernel indeed exhibits spectrallike convergence (saturating at the machine precision).

4.3. Domain with two semi-infinite directions and one fully unbounded BC. Semi-unbounded BCs are finally used. An even symmetry is imposed on the right side of the domain in the $X$ direction, and an odd symmetry is imposed on the left side in 


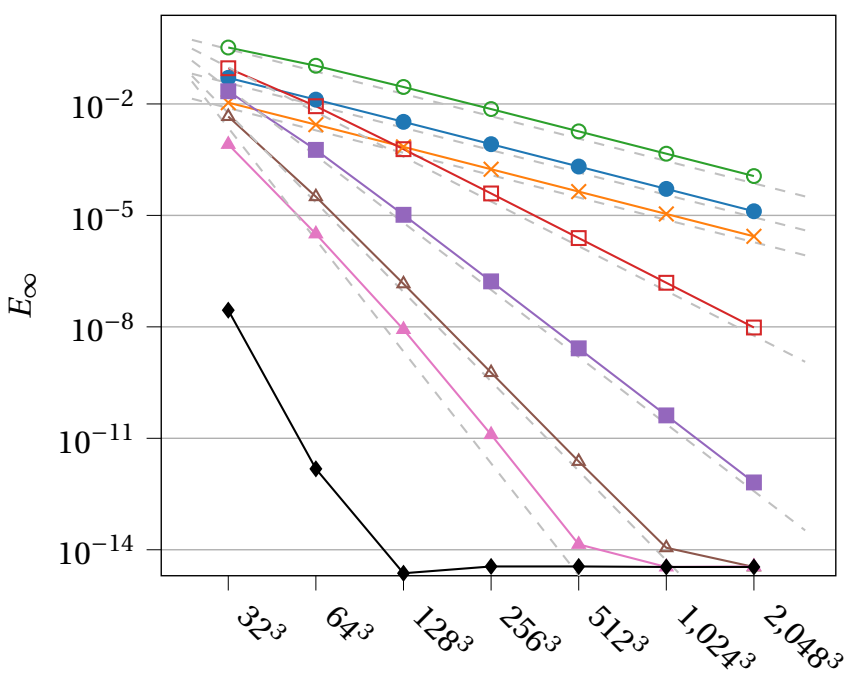

$\mathrm{N}$

Figure 6: Convergence of FLUPS with fully unbounded BCs: $\rightarrow$ CHAT2 $\mathscr{O}\left(h^{2}\right), *$ LGF2 $\mathscr{O}\left(h^{2}\right),-\operatorname{HEJ} 2 \mathscr{O}\left(h^{2}\right),-\square \mathrm{HEJ} 4$ $\mathscr{O}\left(h^{4}\right),-\operatorname{HEJ} 6 \mathscr{O}\left(h^{6}\right), \triangle \operatorname{HEJ} 8 \mathscr{O}\left(h^{8}\right),-\operatorname{HEJ} 10 \mathscr{O}\left(h^{10}\right)$, HEJO spectral-like.

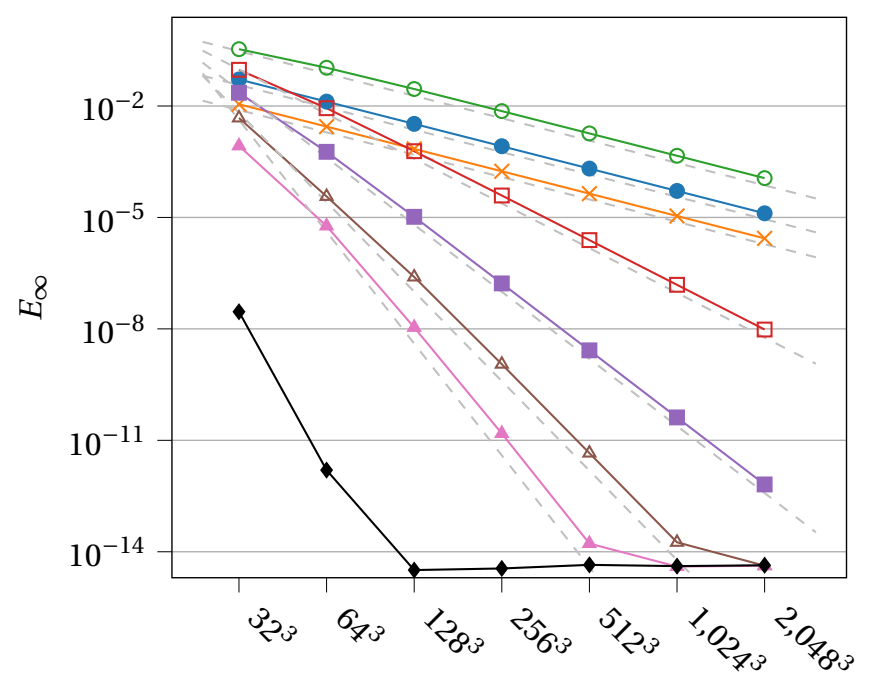

$\mathrm{N}$

Figure 7: Convergence of FLUPS with semi-unbounded BCs: $\rightarrow$ CHAT2 $\mathscr{O}\left(h^{2}\right), *$ LGF2 $\mathscr{O}\left(h^{2}\right),-\mathrm{HEJ} 2 \mathscr{O}\left(h^{2}\right), \square \mathrm{HEJ} 4$ $\mathscr{O}\left(h^{4}\right),-\operatorname{HEJ} 6 \mathscr{O}\left(h^{6}\right), \triangle \operatorname{HEJ} 8 \mathscr{O}\left(h^{8}\right), \triangle \operatorname{HEJ} 10 \mathscr{O}\left(h^{10}\right)$, HEJO spectral-like.

the $Z$ direction. The BCs in the $Y$ direction are kept fully unbounded. The reference solution is

$$
\begin{aligned}
\phi_{r e f}(x, y, z)= & \left.\exp \left(10\left(1-\frac{1}{\left(1-\left(\frac{2 x-1.4}{L}\right)^{2}\right)}\right)\right)+\exp \left(10\left(1-\frac{1}{\left(1-\left(\frac{2 x-2.6}{L}\right)^{2}\right)}\right)\right)\right] \\
& \quad \exp \left(10\left(1-\frac{1}{\left(1-\left(\frac{2 y}{L}-1\right)^{2}\right)}\right)\right)\left[\exp \left(10\left(1-\frac{1}{\left(1-\left(\frac{2 z-0.6}{L}\right)^{2}\right)}\right)\right)-\exp \left(10\left(1-\frac{1}{\left(1-\left(\frac{2 z+0.6}{L}\right)^{2}\right)}\right)\right) .\right.
\end{aligned}
$$

The Green's functions are those of Appendix B.2, and the obtained convergence results are shown in Figure 7 and matches the expected convergence behaviors.

5. Scalability. This section exposes the performance of the non-blocking implementation compared to the all-to-all version and the influence of the partition of the resources between MPI ranks and threads. A speed comparison with the P3DFFT library is also presented.

The mean time required for the execution of the solve operation is tested on three different clusters, listed in Table 1. For the

\begin{tabular}{|c|c|c|c|c|c|c|c|c|}
\hline & Name & Location & Type & $N_{\mathbf{C P U}}$ & CPU & Interconnect & Compiler & MPI \\
\hline 1. & Zenobe $^{a}$ & Belgium & tier-1 & 8208 & Intel Xeon E5-2697v2 & InfiniBand QDR & Intel & Intel \\
\hline 3. & Juwels ${ }^{c}$ & Germany & tier-0 & 122768 & Xeon Platinum 8168 & Infiniband EDR & Intel & Parastation $^{d}$ \\
\hline
\end{tabular}
sake of concision, a selection of plots representative of the behaviors of FLUPS is here presented.

\footnotetext{
${ }^{a}$ Zenobe is in the top 500 supercomputers, more specifications available at www.top500.org/system/178439 (last visited November 2019).

${ }^{b}$ MareNostrum is in the top 500 supercomputers, more specifications available at www.top500.org/system/179067 (last visited November 2019).

${ }^{c}$ Juwels is in the top 500 supercomputers, more specifications available at www.top500.org/system/179424 (last visited October 2019).

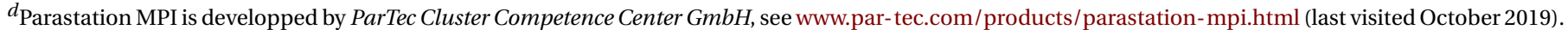

Table 1: List of supercomputers used for testing the scalability of FLUPS.

5.1. Strong scaling. Let $T\left(N_{c}, M\right)$ be the time required to solve a problem of $M$ unknowns using $N_{c}$ cores. Using this definition, the strong efficiency, defined by

$$
\eta_{\text {strong }}=\frac{N_{c}^{\text {ref }} T\left(N_{c}^{\text {ref }}, M^{\text {ref }}\right)}{N_{c} T\left(N_{c}, M^{r e f}\right)}
$$




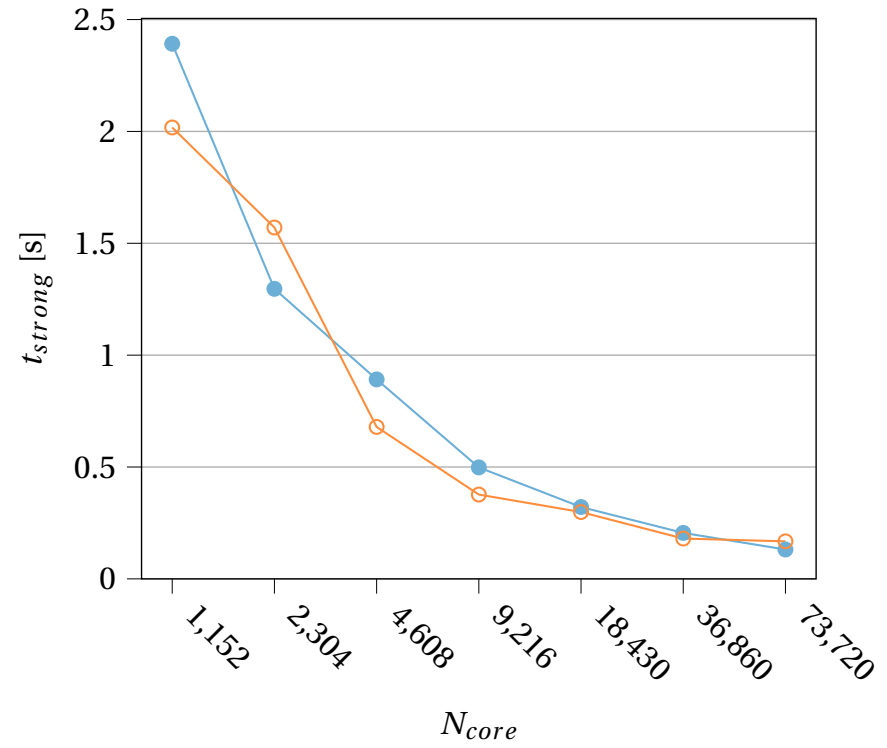

(a) Mean execution time $[s]$ per solve

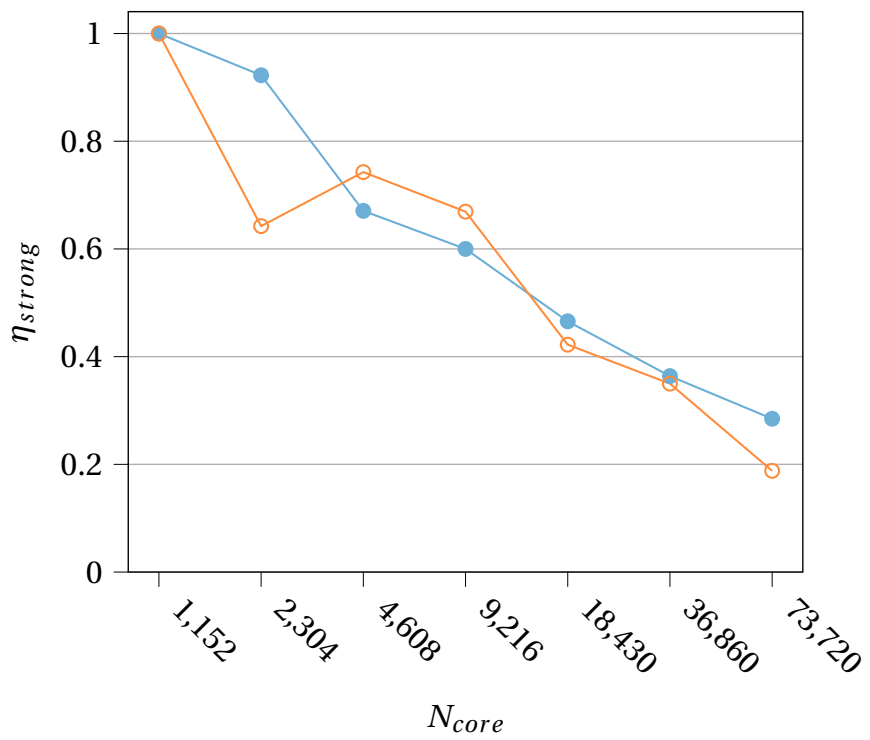

(b) Efficiency

Figure 8: Strong scalability of the solve operation on Juwels, for a fully unbounded problem of size $1152^{3}$ using all-to-all ( $-\bullet-$ ) and non-blocking ( - - ) communications.

describes the ability of the software to decrease the computational time while increasing the number of resources for a given problem size $M_{r e f}$. In this study, the reference size is chosen as $1152^{3}$ cells for a fully unbounded problem, hence leading to a final size of $2304^{3}$ due the domain doubling algorithm. For each data point, the presented timing is obtained as the mean of 20 consecutive solve operations, in order to reduce timing uncertainties and to isolate any external influence of a possible network congestion.

Figure 8a shows the execution time. The non-blocking implementation is usually faster than the all-to-all version, but the advantage significantly shrinks when the number of cores increases. For the largest number of cores, the all-to-all version is even slightly faster. As expected, the gain of starting the memory copies while waiting for the other communications decreases when the number of unknowns per core decreases, and becomes insignificant for a few thousands unknowns per rank. Correspondingly, the size of the MPI messages has become so small that the communication latency globally increases significantly. As even more clearly observed in Figure 8b, FLUPS exhibits the typical strong scalability of communication bounded applications. In the present case, a loss of performance of $70 \%$ is obtained when the number of cores is multiplied by 16 on a unbounded problem of size $1152^{3}$.

5.2. Weak scalability. The weak efficiency, defined as

$$
\eta_{\text {weak }}=\frac{T\left(N_{c}^{r e f}, M^{r e f}\right)}{T\left(N_{c}, \frac{N_{c}}{N_{c}^{r e f}} M^{r e f}\right)},
$$

represents the ability of the software to keep its initial efficiency while increasing the number of cores together with the size of the problem. We highlight that this scalability analysis is the most relevant for the interested user as, practically, the problem size usually scales with the number of resources.

As expected from a communication bounded software, the weak scaling efficiency (Figure 9) decreases with an increasing number of cores, since the number of communications through the network increases as $N_{\text {rank }}^{3 / 2}$, where $N_{\text {rank }}$ is here the number of MPI ranks. This results in the congestion of the network and explains the decreasing efficiency. Indeed, all the clusters used here have a network architecture in tree, with several nodes being connected to switches and switches being connected to racks (unlike BlueGene and Cray computers which feature a multidimensional grid network architecture). Consequently, for calculations on more than 18430 cores, the efficiency is even more reduced, probably due to bottlenecks in this tree network architecture.

The computation time spent in each major step of the solver is shown in Figure 10. As expected, the communication is the most time-expensive part of the solver and drives the scalability, while the other operations almost scale perfectly. Initially, the non-blocking implementation is $10 \%$ faster. For a large number of cores, the all-to-all version becomes more advantageous as it is able to globally balance the communication over the tree network. 


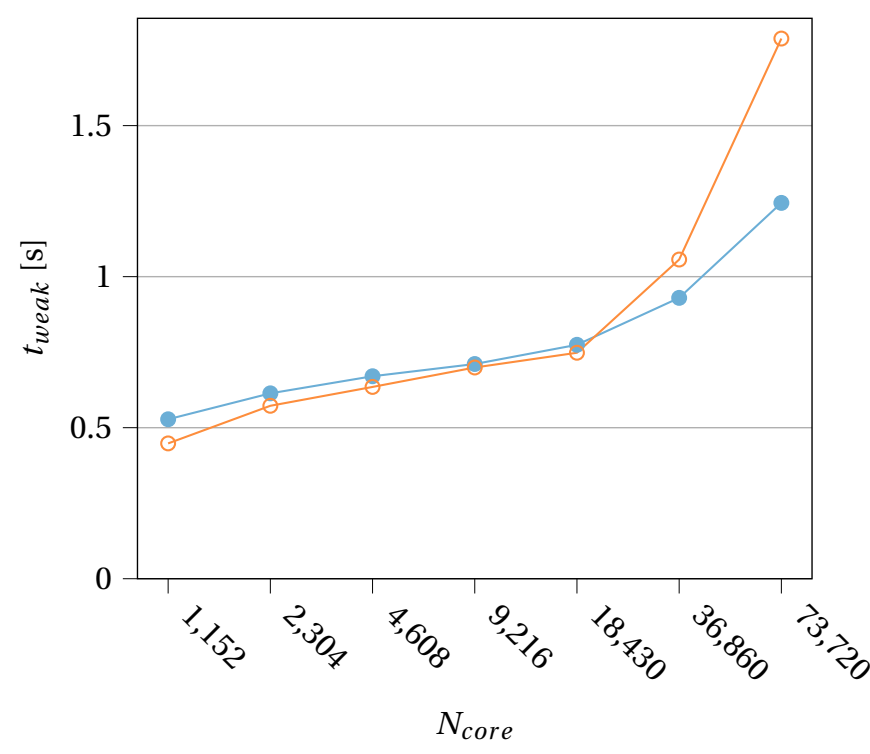

(a) Mean execution time

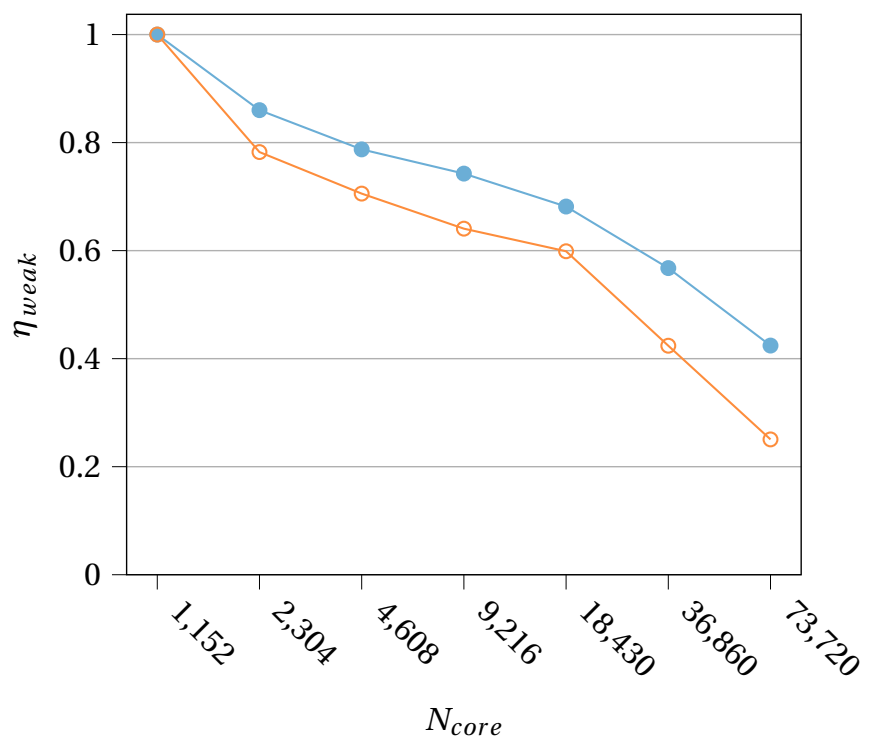

(b) Efficiency

Figure 9: Weak scaling efficiency of the two implementations on Juwels, for a fully unbounded problem and $64^{3}$ unknowns per core using all-to-all ( $-\infty)$ and non-blocking $(-\infty)$ communications.

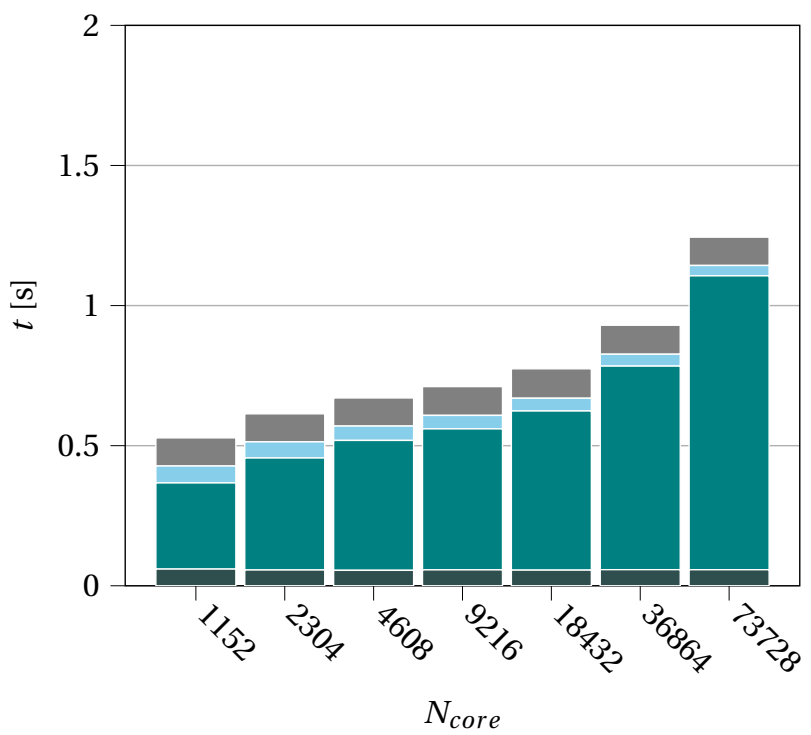

(a) All-to-all communications

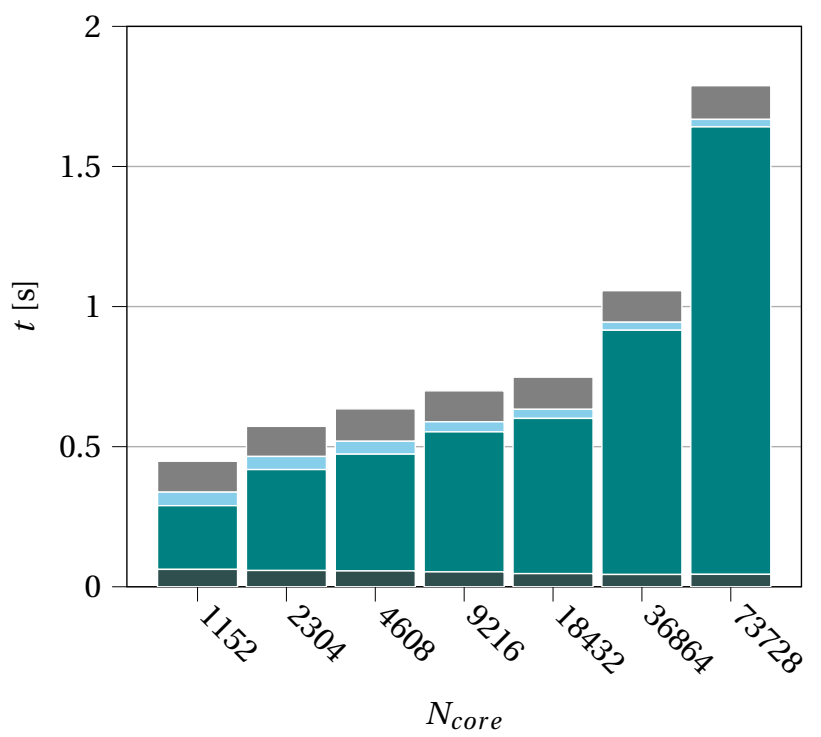

(b) Non-blocking communications

Figure 10: Time spent in each part of the 'solve' operation using $64^{3}$ unknowns by core, on Juwels. From bottom to top, the shaded areas correspond to the computation of the 1D Fourier transforms $(\square)$, the time spent in the communication waiting for the data to be transferred ( $\square$ ), the data transfer from the memory to the send buffers and from the receive buffers to the transposed memory ( $\square$ ) and miscellaneous operations ( $\square$ ). The latter includes, among others, the multiplication in Fourier space for the convolution and the reset of the buffers to zero.

A similar communication-bounded behavior is also observed when increasing the computational load to $128^{3}$ unknowns per core (see Figure 11). However, for this size, the non-blocking communication approach is clearly faster by $\pm 20-25 \%$ compared to the all-to-all approach. This is due to the overlap between the communication and the copy of the data to the transposed memory. This technique turns out to be beneficial only when the number of data to copy is large enough to compensate the less efficient communication, compared to an all-to-all implementation.

Incidentally, one could argue that other computational operations could possibly be executed during the communication. For 


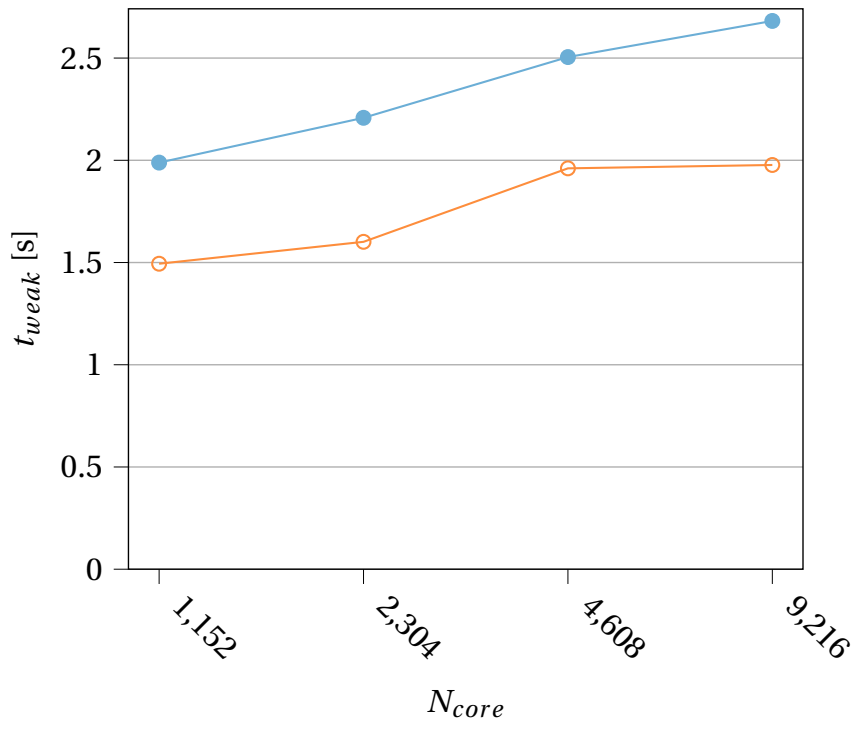

(a) Mean execution time

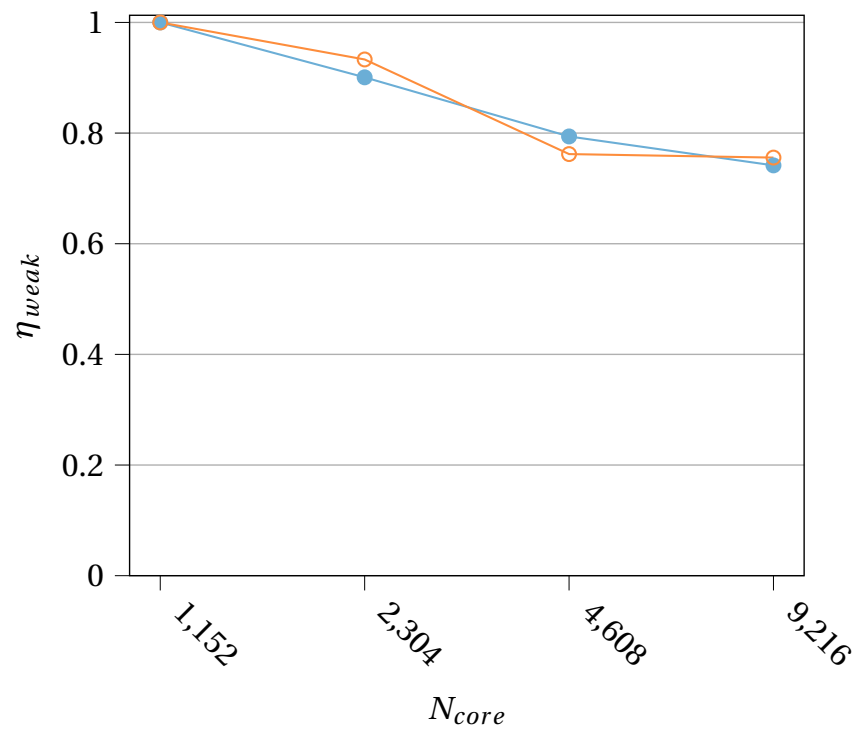

(b) Efficiency

Figure 11: Weak scalability on MareNostrum with all-to-all ( $-\bullet$ ) and non-blocking ( - ) communications, using 1 thread per rank and $128^{3}$ unknowns per core .

instance, the computation of the FFTs could start as soon as the first complete row of data in the pencil has been received. However, since the FFTs represent less than $10 \%$ of the computation time, this would likely not significantly improve the computational efficiency, while greatly increasing the implementation complexity. Even worse, it would overwhelm the network with a lot of very small messages, hence slowing down the solver.

5.3. Hybrid MPI/OpenMP parallel performance. We compare the pure MPI and a hybrid MPI-OpenMP layout (without hyperthreading). The former defines as many MPI ranks as the number of available cores, $N_{\text {rank }}=N_{\text {core }}$. In the latter, the cores that belong to the same socket can be grouped and execute a chosen number of threads, such that $N_{\text {rank }}=N_{\text {core }} / N_{\text {thread }}$. (We use the MPI_THREAD_FUNNELED mode in order to mitigate the communication cost.)

Let us first examine the expected influence of the addition of threads. On theoretical grounds, the simplified PlogP model [1] decomposes the communication time into a constant overhead, a linear dependency on the number of messages to send and a linear dependency on the message size. In the case of a pure MPI layout, one core sends and receives $P=\sqrt{N_{\text {core }}}$ messages, each of size $N_{\text {data }}$, hence leading to a communication time of

$$
T_{\mathrm{comm}}^{M P I}=\alpha+P g+N_{\text {data }} G(P)
$$

where $g$ is the gap between messages (expressed in $[s]$ ) and $G$ is the gap per byte for long messages $[s / B]$. Grouping $N_{\text {thread }}$ cores under the same MPI rank will decrease the number of sent messages from $P$ to $P / N_{\text {thread }}$, which results in a communication time given by

$$
T_{\text {comm }}^{\text {hybrid }}=\alpha+P / N_{\text {thread }} g+N_{\text {thread }} N_{\text {data }} G\left(P / N_{\text {thread }}\right) .
$$

The increase in size of the messages has a beneficial effect through the second term of (5.4), and a detrimental effect through the last term. The latter might however be compensated by the increased bandwidth (i.e., the rate at which data is transferred, equivalent to $1 / G$ ) generally observed for larger messages. Besides, the use of threads within a single MPI rank also adds a nonnegligible overhead, due to the thread management in the copy and the shuffle operations. Due to these antagonist effects, the use of threads may result in either improved or deteriorated performances, and dedicated tests must be performed ${ }^{3}$. Notice that the computational cost associated with the FFT remains unchanged in a multithreaded environment, as the pencils are distributed among the threads and each thread treats them sequentially using single-thread FFTs.

As observed in the weak scaling presented in Figure 12, only the small number of cores benefits from an acceleration when using 4 threads and all-to-all communications, while it is significantly slower on large partitions. Indeed, in the present all-to-all

\footnotetext{
${ }^{3}$ Even though the general concepts presented in this paragraph are true, the actual performance may vary depending on the targeted network architecture. In order to ease the determination by the user of the optimal thread setting for the machine he plans to use, we provide guidelines to run dedicated tests in the code documentation.
} 


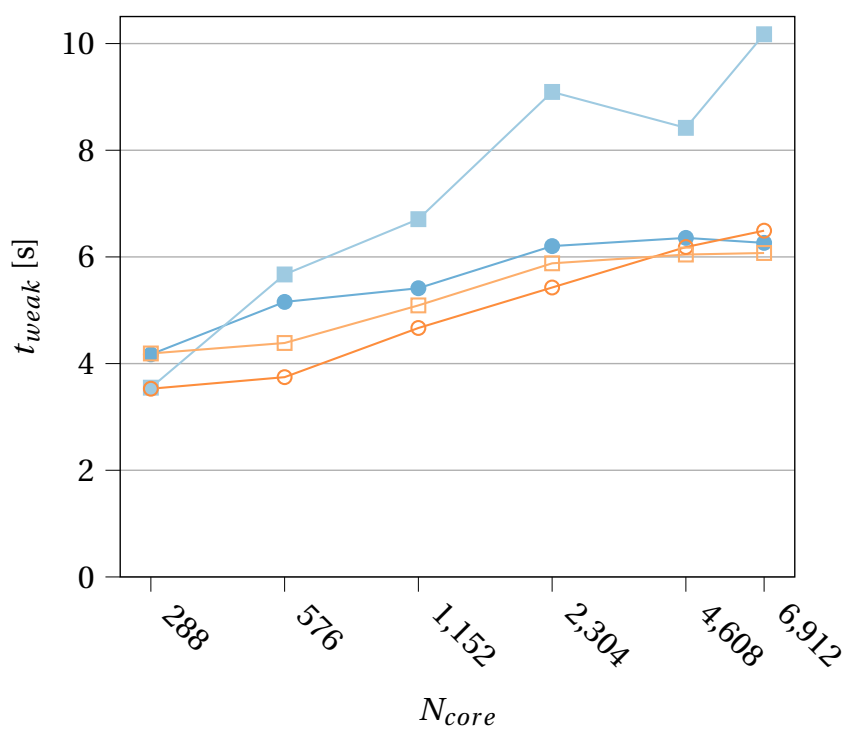

(a) Mean execution time

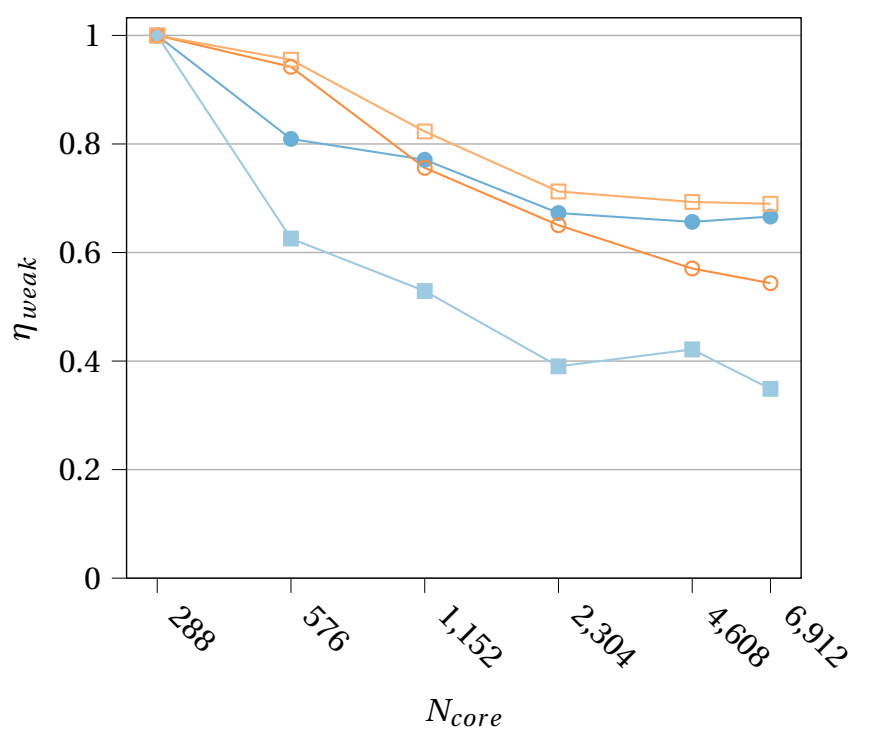

(b) Efficiency

Figure 12: Weak scalability on Zenobe with 1 thread per rank (all-to-all: - - - non-blocking: $-\bullet$ ) and 4 threads per rank (all-to-all: - , non-blocking: $-\square)$, using $128^{3}$ unknowns per core.

implementation, the communication and the copy are sequential. Hence, the copy overhead remains the same, but the communication cost drastically increases with the number of cores. Indeed, the communication time is driven by the cost associated with the message length, $N_{\text {thread }} N_{\text {data }} G$, which is here not compensated by a gain in bandwidth.

However, in the non-blocking approach, the communication overlaps with the copy and with the shuffle operations. Even though the time-to-solution slightly increases for small $N_{\text {core }}$ with respect to the non-threaded implementation (attributed to the overhead of threads), the non-scalable communication times are thus partially hidden behind more scalable copy and shuffle operations, which explains the better scalability obtained with the hybrid MPI-OpenMP non-blocking approach. Consequently, the threaded execution becomes faster than the non-threaded one at high $N_{\text {core }}$. Furthermore, the non-blocking version with threads is mostly faster than the all-to-all version without threads.

Finally, the comparison of the results obtained with different clusters reveals that the scalability is strongly dependent on the running environment, with a difference in efficiency larger than $10 \%$ for large sizes, as seen in Figure 13 . The driving factors are the different interconnects and the various MPI implementations.

5.4. Comparison with P3DFFT++. A campaign of computations was dedicated to compare the performances of the P3DFFT library $^{4}$ V3 (also known as P3DFFT++) with those of FLUPS (for the 3D transform only). To this end, a single executable is created, in which each library is used to perform the same fully periodic 3D FFT. As opposed to the previous section, the starting topology is here already set up with a pencil decomposition, such that the execution will only involve two topology switches and three computations of 1D FFT. Both libraries use the same binary from the compilation of FFTW. They also both use the FFTW_MEASURE option when creating 1D FFT plans, such that the difference in timing only originates in the implementation of the 3D FFT, specific to FLUPS and P3DFFT.

Similarly to the weak scaling study, a resolution of $128^{3}$ per core was selected, and the number of cores was increased (using 1 thread in FLUPS). The results were obtained on Zenobe and are shown in Figure 14.

On average, FLUPS is roughly $20 \%$ faster than P3DFFT++, and exhibits a similar scalability. For this comparison, the all-to-all version of FLUPS was used. An even larger difference is thus to be expected with the non-blocking implementation.

6. Conclusions. The Fourier-based Library of Unbounded Poisson Solvers (FLUPS) enables the fast resolution of the Poisson equation on 2D rectangle and 3D parallelepiped-like domains with uniform resolution and with arbitrary boundary conditions on each boundary. The library features a collection of solvers using a Green's function of various types, and with a verified convergence order from 2 to spectral-like, to solve the Poisson equation. The convolution of the Green's function with the RHS of the Poisson equation is performed in Fourier space. To this end, data are transformed using fast Fourier transforms performed in 1D pencils and transposed efficiently.

The mathematical background for the resolution of the Poisson equation with the various boundary conditions was exposed,

\footnotetext{
${ }^{4}$ The latest version of P3DFFT V3 available at the time of this study was used, with git SHA 2150652831daab38b1028d16db7e4c7318df1133 from github.com/ sdsc/p3dfft.3.
} 


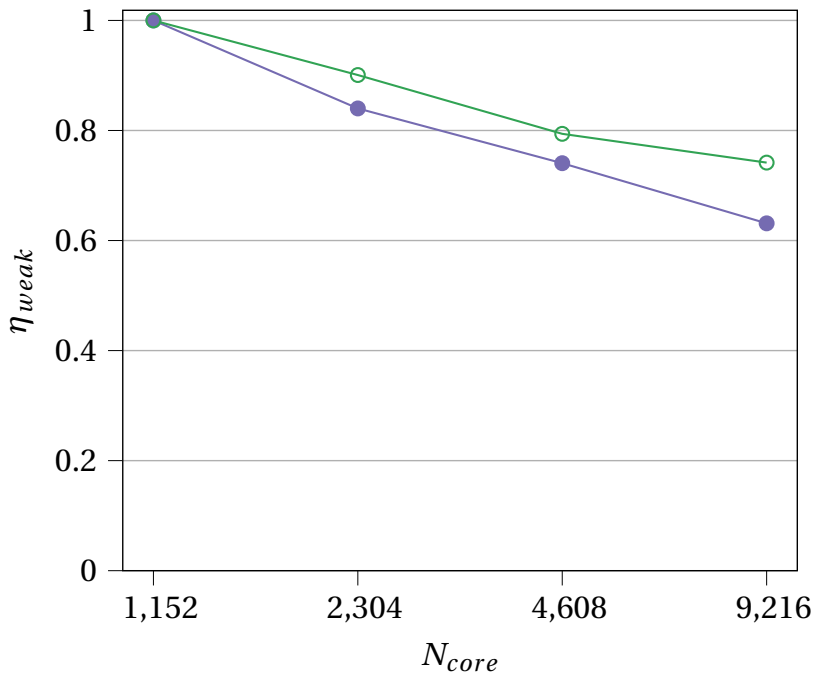

Figure 13: Weak scaling efficiency of the all-to-all implementation on Juwels ( $-\bullet$ ) and MareNostrum ( $-\bullet$ ), for a fully unbounded problem and $128^{3}$ unknowns per core.

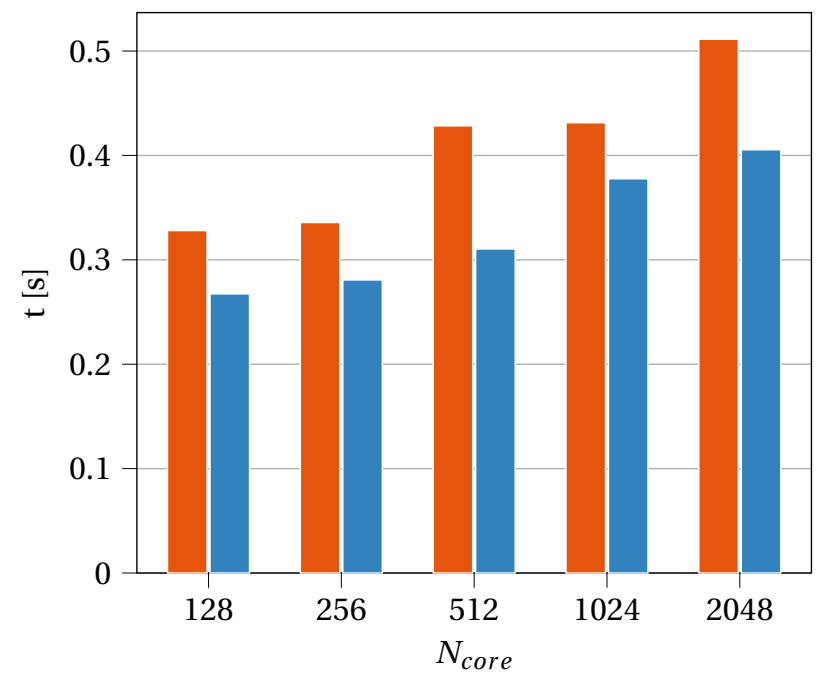

Figure 14: Wall time for a 3D FFT, using the all-to-all version of FLUPS ( $\square$ ) and P3DFFT++ ( $\square$ ), executed on Zenobe, using $128^{3}$ unknowns per core.

including the newly enabled semi-unbounded directions. In particular, the numerical treatment of data required for the RHS and for the Green's function was detailed in all cases. The particularities of the numerical implementation were also addressed, especially related to the implementation of the 3D FFT. The comparison between two communication methods showed that pointto-point non-blocking MPI send-receive instructions lead to a faster execution when they are interlaced with memory copy operations, compared to regular all-to-all directives. Under some conditions on the communication latency and on the size of the MPI messages, the use of threads can even further improve the overall execution time. The scalability of the library was measured on current top world-class supercomputers with tree-like network architectures. It features the behavior typical of communicationbounded software: when multiplying the number of cores by 16 , the strong efficiency drops by $70 \%$ (for a problem of size $1152^{3}$ ) and the weak efficiency by $60 \%$ (for $64^{3}$ unknowns per core). Still, FLUPS has been demonstrated to be roughly $20 \%$ faster than a reference third-party package for 3D FFTs.

The final implementation hence exhibits encouraging performances, propitious to massively parallel computational architectures, which proves that FLUPS is now ready for production use.

Acknowledgements. The authors would like to acknowledge the insightful discussions with Prof. G. Winckelmans, Dr. M. Duponcheel and Dr. J. Lambrechts (UCLouvain, Belgium).

D.-G. Caprace is funded by an Aspirant fellowship from the Fonds de la Recherche Scientifique de Belgique, FR.S.-FNRS. Computational resources have been provided by the Consortium des Équipements de Calcul Intensif (CÉCI), funded by the Fonds de la Recherche Scientifique de Belgique (F.R.S.-FNRS) under Grant $n^{\circ} 2.5020 .11$ and by the Walloon Region. The present research also benefited from computational resources made available on the Tier-1 supercomputer of the Federation Wallonie-Bruxelles, infrastructure funded by the Walloon Region under the grant agreement $n^{\circ} 1117545$. Finally, we acknowledge PRACE for awarding us access to MareNostrum at Barcelona Supercomputing Center (BSC), Spain; and JUWELS at GCS@FZJ, Germany.

\section{References.}

[1] A. Alexandrov, M. F. Ionescu, K. E. Schauser, and C. Scheiman, Loggp: Incorporating long messages into the logp model for parallel computation, Journal of Parallel and Distributed Computing, 44 (1997), pp. 71-79, https://doi.org/https://doi.org/10.1006/jpdc.1997.1346, http://www.sciencedirect.com/ science/article/pii/S0743731597913460.

[2] C. R. ANDERSON, High order expanding domain methods for the solution of poisson's equation in infinite domains, Journal of Computational Physics, 314 (2016), pp. 194 - 205, https://doi.org/https://doi.org/10.1016/j.jcp.2016.02.074, http://www.sciencedirect.com/science/article/pii/S0021999116001613.

[3] J. C. BOWMAN AND M. ROBERTS, Adaptive matrix transpose algorithms for distributed multicore processors, in Interdisciplinary Topics in Applied Mathematics, Modeling and Computational Science, Springer, 2015, pp. 97-103.

[4] P. Chatelain And P. Koumoutsakos, A Fourier-based elliptic solver for vortical flows with periodic and unbounded directions, Journal of Computational Physics, 229 (2010), pp. 2425-2431.

[5] P. Chatelain AND A. LeONARD, Isotropic compact interpolation schemes for particle methods, Journal of Computational Physics, 227 (2008), pp. 3244 - 3259, https://doi.org/http://dx.doi.org/10.1016/j.jcp.2007.11.039.

[6] A. G. Chatterjee, M. K. Verma, A. Kumar, R. Samtaney, B. Hadri, and R. Khurram, Scaling of a fast fourier transform and a pseudo-spectral fluid solver up to 196608 cores, Journal of Parallel and Distributed Computing, 113 (2018), pp. 77 - 91, https://doi.org/https://doi.org/10.1016/j.jpdc.2017.10.014, http://www.sciencedirect.com/science/article/pii/S0743731517302903.

[7] P. CostA, A fft-based finite-difference solver for massively-parallel direct numerical simulations of turbulent flows, Computers \& Mathematics with Applications, 76 (2018), pp. 1853 - 1862, https://doi.org/https://doi.org/10.1016/j.camwa.2018.07.034, http://www.sciencedirect.com/science/article/pii/ S089812211830405X.

[8] L. Dalcin, M. Mortensen, And D. E. Keyes, Fast parallel multidimensional fft using advanced mpi, Journal of Parallel and Distributed Computing, 128 
(2019), pp. 137 - 150, https://doi.org/https://doi.org/10.1016/j.jpdc.2019.02.006, http://www.sciencedirect.com/science/article/pii/S074373151830306X.

[9] B. Dorschner, K. Yu, G. Mengaldo, and T. Colonius, A fast multi-resolution lattice green's function method for elliptic difference equations, Journal of Computational Physics, 407 (2020), p. 109270.

[10] M. Frigo And S. G. Johnson, The Design and Implementation of FFTW3, Proceedings of the IEEE, 93 (2005), pp. 216-231. Special issue on Program Generation, Optimization, and Platform Adaptation.

[11] V. FuKA, Poisfft - a free parallel fast poisson solver, Applied Mathematics and Computation, 267 (2015), pp. 356 - 364, https://doi.org/https://doi.org/10.1016/ j.amc.2015.03.011, http://www.sciencedirect.com/science/article/pii/S0096300315003057. The Fourth European Seminar on Computing (ESCO 2014).

[12] A. Gholami, D. Malhotra, H. Sundar, AND G. Biros, FFt, Fmm, or Multigrid? A comparative Study of State-Of-the-Art Poisson Solvers for Uniform and Nonuniform Grids in the Unit Cube, SIAM Journal on Scientific Computing, 38 (2016), pp. C280-C306.

[13] T. Gillis, G. Winckelmans, and P. Chatelain, Fast immersed interface Poisson solver for 3D unbounded problems around arbitrary geometries, Journal of Computational Physics, 354 (2018), pp. 403 - 416, https://doi.org/https://doi.org/10.1016/j.jcp.2017.10.042.

[14] A. Gillman AND P. Martinsson, Fast and accurate numerical methods for solving elliptic difference equations defined on lattices, Journal of Computational Physics, 229 (2010), pp. 9026 - 9041, https://doi.org/http://dx.doi.org/10.1016/j.jcp.2010.07.024.

[15] A. Gillman And P. Martinsson, A fast solver for Poisson problems on infinite regular lattices, Journal of Computational and Applied Mathematics, 258 (2014), pp. 42 - 56, https://doi.org/http://dx.doi.org/10.1016/j.cam.2013.09.003.

[16] L. Greengard, S. JIANG, AND Y. Zhang, The anisotropic truncated kernel method for convolution with free-space green's functions, SIAM Journal on Scientific Computing, 40 (2018), pp. A3733-A3754.

[17] L. Greengard and V. Rokhlin, A fast Algorithm for Particle Simulations, Journal of Computational Physics, 135 (1997), pp. 280 - 292.

[18] M. Hejlesen, J. Rasmussen, P. Chatelain, And J. Walther, A high order solver for the unbounded Poisson equation, Journal of Computational Physics, 252 (2013), pp. 458-467, https://doi.org/10.1016/j.jcp.2013.05.050.

[19] M. M. Hejlesen, G. WincKelmans, AND J. H. Walther, Non-singular green's functions for the unbounded poisson equation in one, two and three dimensions, Applied Mathematics Letters, 89 (2019), pp. 28-34, https://doi.org/https://doi.org/10.1016/j.aml.2018.09.012, http://www.sciencedirect.com/science/ article/pii/S0893965918303264.

[20] R. Hockney And J. EASTwood, Computer Simulation using Particles, Taylor \& Francis, Inc. Bristol, PA, USA, 1988.

[21] R. W. HockNEY, A fast direct solution of poisson's equation using fourier analysis, Journal of the Association for Computing Machinery, 12 (1965), pp. $95-113$.

[22] H. Ibeid, L. Olson, AND W. Gropp, Fft, fmm, and multigrid on the road to exascale: Performance challenges and opportunities, Journal of Parallel and Distributed Computing, 136 (2020), pp. 63-74.

[23] J. L. Jodra, I. Gurrutxaga, J. Muguerza, AND A. Yera, Solving poisson's equation using fft in a gpu cluster, Journal of Parallel and Distributed Computing, 102 (2017), pp. 28 - 36, https://doi.org/https://doi.org/10.1016/j.jpdc.2016.09.004, http://www.sciencedirect.com/science/article/pii/S0743731516301678.

[24] S. Liska AND T. Colonius, A parallel fast multipole method for elliptic difference equations, Journal of Computational Physics, 278 (2014), pp. 76 - 91 , https://doi.org/http://dx.doi.org/10.1016/j.jcp.2014.07.048.

[25] P.-G. Martinsson and G. J. Rodin, Asymptotic expansion of lattice Green's function, Proceedings: Mathematical, Physical and Engineering Sciences, 458 (2002), pp. 2609-2622.

[26] D. Pekurovsky, P3dff: A framework for parallel computations of fourier transforms in three dimensions, SIAM Journal on Scientific Computing, 34 (2012), pp. C.192-C.209, https://search-proquest-com.proxy.bib.ucl.ac.be:2443/docview/1033558975?accountid=12156. Copyright - () 2012, Society for Industrial and Applied Mathematics; Dernière mise à jour - 2012-09-17.

[27] M. PIPPIG, Pfft: An extension of fftw to massively parallel architectures, SIAM Journal on Scientific Computing, 35 (2013), pp. C213-C236, https://doi.org/10. 1137/120885887, https://doi.org/10.1137/120885887.

[28] J. QIANG, A fast parallel 3d poisson solver with longitudinal periodic and transverse open boundary conditions for space-charge simulations, Computer Physics Communications, 219 (2017), pp. 255 - 260, https://doi.org/https://doi.org/10.1016/j.cpc.2017.06.002, http://www.sciencedirect.com/science/article/pii/ S0010465517301844.

[29] I. F. Sbalzarini, J. H. Walther, B. Polasek, P. Chatelain, M. Bergdorf, S. E. Hieber, E. M. Kotsalis, and P. Koumoutsakos, a software framework for the portable parallelization of particle-mesh simulations, 2006.

[30] H. J. SPIETZ, M. M. HEJLESEN, AND J. H. WALTHER, A regularization method for solving the poisson equation for mixed unbounded-periodic domains, Journal of Computational Physics, 356 (2018), pp. 439 - 447, https://doi.org/https://doi.org/10.1016/j.jcp.2017.12.018, http://www.sciencedirect.com/science/ article/pii/S0021999117309038.

[31] A. Sunderland, S. Pickles, M. Nikolic, A. Jovic, J. Jakic, V. Slavnic, I. Girotto, P. Nash, and M. Lysaght, An analysis of fft performance in prace application codes, tech. report, PRACE, 2012.

[32] U. Trottenberg, C. Oosterlee, and A. Schuller, Multigrid, Academic Press, 2000.

[33] F. Vico, L. Greengard, and M. Ferrando, Fast convolution with free-space Green's functions, Journal of Computational Physics, 323 (2016), pp. 191 - 203, https://doi.org/http://dx.doi.org/10.1016/j.jcp.2016.07.028. 


\section{Appendix A. Analytical expressions of the Gaussian regularisation functions.}

The regularization functions considered in this work are based on radially symmetric functions $\zeta_{m}(\rho)$,

$$
\zeta_{\varepsilon, m}(r)=\frac{1}{\varepsilon^{d}} \zeta_{m}\left(\frac{r}{\varepsilon}\right)
$$

where $d$ is the dimensionality of the space. For a given order $m, \zeta_{\varepsilon}^{m}$ satisfies the $m$ first moments, that is, $\forall \beta \in\{0, \ldots, m-2\}$,

$$
0^{\beta}= \begin{cases}2 \int_{0}^{\infty} r^{\beta} \zeta_{\varepsilon}^{m}(r) d r & \text { in } 1 \mathrm{D}, \\ 2 \pi \int_{0}^{\infty} r^{\beta} \zeta_{\varepsilon}^{m}(r) r d r & \text { in } 2 \mathrm{D}, \\ 4 \pi \int_{0}^{\infty} r^{\beta} \zeta_{\varepsilon}^{m}(r) r^{2} d r & \text { in 3D, }\end{cases}
$$

Notice that the constraint is always satisfied for odd $\beta$ as the function $\zeta^{m}$ is radially symmetric.

The Gaussian regularization functions were extensively used in [18] and by co-authors in other works, and their expressions are here reproduced for the sake of completeness. In that particular case,

$$
\zeta_{m}(\rho)=P_{m}(\rho) e^{\left(\frac{-\rho^{2}}{2}\right)},
$$

where $P_{m}(\rho)=a_{1}+a_{2} \rho^{2}+\cdots+a_{m / 2} \rho^{m-2}$. For a given $m$, the fulfilment of (A.2) reduces to solving a system of $m / 2$ equations for the coefficients $a_{i}$. Alternatively, $\zeta_{m}$ can be obtained as the result of a recursive procedure [5]. For example, in 1D,

$$
\begin{gathered}
P_{2}(\rho)=1, \quad P_{4}(\rho)=\frac{1}{2}\left(3-\rho^{2}\right), \quad P_{6}(\rho)=\frac{1}{8}\left(15-10 \rho^{2}+\rho^{4}\right), \quad P_{8}(\rho)=\frac{1}{48}\left(105-105 \rho^{2}+21 \rho^{4}-\rho^{6}\right), \\
P_{10}(\rho)=\frac{1}{384}\left(945-1260 \rho^{2}+378 \rho^{4}-36 \rho^{6}+\rho^{10}\right)
\end{gathered}
$$

It is also convenient to express the Fourier transform of (A.3),

$$
\hat{\zeta}_{m}(s)=D_{m}(s) e^{-\frac{s^{2}}{2}}
$$

where $D_{m}(s)=\sum_{n=0}^{m / 2-1} \frac{\left(s^{2} / 2\right)^{n}}{n !}$.

\section{Appendix B. Analytical expressions of the Green's functions in 3D.}

In this section, the expression of the Green's kernels $G$ used in FLUPS to solve the Poisson equation, $\nabla^{2} \phi=f$, is reproduced. The solution is obtained as $\phi=G * f$.

\section{B.1. 3 directions unbounded.}

B.1.1. Singular. The exact solution, and the cell-averaged value replacement, reproduced from [4], are, respectively,

$$
G(\mathbf{x})=-\frac{1}{4 \pi|\mathbf{x}|} \quad \text { and } \quad G(\mathbf{0})=-\frac{1}{2}\left(\frac{1}{2 \pi} \frac{3 h^{3}}{2}\right)^{\frac{2}{3}}
$$

B.1.2. Regularized. The regularized Green's functions, reproduced from [18], use $\rho=\frac{|\mathbf{x}|}{\varepsilon}$, such that

$$
G_{m}(|\mathbf{x}|)=-\frac{1}{4 \pi|\mathbf{x}|}\left(Q_{m}(\rho) e^{-\frac{\rho^{2}}{2}}+\operatorname{erf}\left(\frac{\rho}{\sqrt{2}}\right)\right),
$$

using the conventional definition of $\operatorname{erf}(z)=\frac{2}{\sqrt{\pi}} \int_{0}^{z} e^{-q^{2}} d q$, and where $Q_{m}$ is given by

$$
\begin{gathered}
Q_{2}(\rho)=0, \quad Q_{4}(\rho)=\frac{1}{\sqrt{2 \pi}}(\rho), \quad Q_{6}(\rho)=\frac{1}{\sqrt{2 \pi}}\left(\frac{7}{4} \rho-\frac{1}{4} \rho^{3}\right), \quad Q_{8}(\rho)=\frac{1}{\sqrt{2 \pi}}\left(\frac{19}{8} \rho-\frac{2}{3} \rho^{3}+\frac{1}{24} \rho^{5}\right), \\
Q_{10}(\rho)=\frac{1}{\sqrt{2 \pi}}\left(\frac{187}{64} \rho-\frac{233}{192} \rho^{3}+\frac{29}{192} \rho^{5}-\frac{1}{192} \rho^{7}\right) .
\end{gathered}
$$

The limit for $|\mathbf{x}| \longrightarrow 0$ is given by

$$
G_{2}(\mathbf{0})=-\frac{1}{4} \frac{\sqrt{2}}{\pi^{3 / 2} \epsilon}, \quad G_{4}(\mathbf{0})=-\frac{3}{8} \frac{\sqrt{2}}{\pi^{3 / 2} \epsilon}, \quad G_{6}(\mathbf{0})=-\frac{15}{32} \frac{\sqrt{2}}{\pi^{3 / 2} \epsilon}, \quad G_{8}(\mathbf{0})=-\frac{35}{64} \frac{\sqrt{2}}{\pi^{3 / 2} \epsilon}, \quad G_{10}(\mathbf{0})=-\frac{315}{512} \frac{\sqrt{2}}{\pi^{3 / 2} \epsilon} .
$$

A generalization to spectrally convergent kernels was proposed in [19], here noted as $G_{\infty}$, which leads to

$$
G_{\infty}(|\mathbf{x}|)=\frac{1}{2 \pi^{2} \sigma} \frac{\operatorname{Si}(\rho)}{\rho}, \quad G_{\infty}(\mathbf{0})=-\frac{1}{2 \pi^{2} \sigma}
$$

where $\sigma=\frac{h}{\pi}$ is the spectral cutoff, and $\operatorname{Si}(x) \triangleq \int_{0}^{x} \frac{\sin (t)}{t} d t$ is the sine integral function. 
B.1.3. LGF. The lattice Green's function is defined as

$$
G_{h}(\mathbf{x})=\frac{1}{(2 \pi)^{3}} \iiint_{-\pi / h}^{\pi / h} \frac{h^{2} \exp (-\mathbf{i} \mathbf{x} \cdot \mathbf{k})}{4 \sin ^{2}\left(k_{x} h / 2\right)+4 \sin ^{2}\left(k_{y} h / 2\right)+4 \sin ^{2}\left(k_{z} h / 2\right)} d \mathbf{k},
$$

where $h$ is the uniform grid size and $k_{x, y, z} \in\left[\frac{-\pi}{h}, \frac{\pi}{h}\right]$.

Here instead, it is evaluated using the indexed form, $G(\mathbf{x}) \triangleq \frac{1}{h} \Theta(\mathbf{m})$, where $\mathbf{m}=\frac{\mathbf{x}}{h}=\left\{m_{x} ; m_{y} ; m_{z}\right\}, \boldsymbol{\xi}=\mathbf{k} h$ and

$$
\Theta(\mathbf{m})=\frac{1}{(2 \pi)^{3}} \iiint_{-\pi}^{\pi} \frac{\exp (-\mathbf{i m} \cdot \boldsymbol{\xi})}{4 \sin ^{2}\left(\xi_{x} / 2\right)+4 \sin ^{2}\left(\xi_{y} / 2\right)+4 \sin ^{2}\left(\xi_{z} / 2\right)} d \boldsymbol{\xi}=-\int_{0}^{\infty} e^{-6 t} I_{m_{x}}(2 t) I_{m_{y}}(2 t) I_{m_{z}}(2 t) d t
$$

The integral (B.7) is precomputed using its second form ${ }^{5}$ and stored for every point satisfying $\max \left[m_{x}, m_{y}, m_{z}\right]<64$. For every other location, we use the following expansion such that the estimated error is lower that the machine precision [13], i.e. with an estimated error of $64^{-9} \approx 10^{-17}$,

(B.8)

$$
\begin{aligned}
& \Theta(\mathbf{m})=\frac{-1}{4 \pi|\mathbf{m}|} \quad-\frac{1}{16 \pi|\mathbf{m}|^{7}}\left[\quad m_{x}^{4}+m_{y}^{4}+m_{z}^{4}-3 m_{x}^{2} m_{y}^{2}-3 m_{x}^{2} m_{z}^{2}-3 m_{y}^{2} m_{z}^{2}\right] \\
& -\frac{1}{128 \pi|\mathbf{m}|^{13}}\left[\left(m_{x}^{8}+m_{y}^{8}+m_{z}^{8}\right)-244\left(m_{x}^{6}\left(m_{y}^{2}+m_{z}^{2}\right)+m_{y}^{6}\left(m_{x}^{2}+m_{z}^{2}\right)+m_{z}^{6}\left(m_{x}^{2}+m_{y}^{2}\right)\right)\right. \\
& \left.-228 m_{x}^{2} m_{y}^{2} m_{z}^{2}|\mathbf{m}|^{2}+621\left(m_{x}^{4} m_{y}^{4}+m_{y}^{4} m_{z}^{4}+m_{x}^{4} m_{z}^{4}\right)\right] \\
& -\frac{1}{2048 \pi|\mathbf{m}|^{19}}\left[2588\left(m_{x}^{12}+m_{y}^{12}+m_{z}^{12}\right)\right. \\
& -65676\left(m_{x}^{10} m_{y}^{2}+m_{x}^{2} m_{y}^{10}+m_{x}^{10} m_{z}^{2}+m_{x}^{2} m_{z}^{10}+m_{y}^{10} m_{z}^{2}+m_{y}^{2} m_{z}^{10}\right) \\
& +426144\left(m_{x}^{8} m_{y}^{4}+m_{x}^{4} m_{y}^{8}+m_{x}^{8} m_{z}^{4}+m_{x}^{4} m_{z}^{8}+m_{y}^{8} m_{z}^{4}+m_{y}^{4} m_{z}^{8}\right) \\
& -712884\left(m_{x}^{6} m_{y}^{6}+m_{x}^{6} m_{z}^{6}+m_{y}^{6} m_{z}^{6}\right) \\
& -62892\left(m_{x}^{8} m_{y}^{2} m_{z}^{2}+m_{x}^{2} m_{y}^{8} m_{z}^{2}+m_{x}^{2} m_{y}^{2} m_{z}^{8}\right) \\
& -297876\left(m_{x}^{6} m_{y}^{4} m_{z}^{2}+m_{x}^{6} m_{y}^{2} m_{z}^{4}+m_{x}^{4} m_{y}^{6} m_{z}^{2}+m_{x}^{2} m_{y}^{6} m_{z}^{4}+m_{x}^{2} m_{y}^{4} m_{z}^{6}+m_{x}^{4} m_{y}^{2} m_{z}^{6}\right) \\
& \left.+2507340\left(m_{x}^{4} m_{y}^{4} m_{z}^{4}\right)\right]+\mathscr{O}\left(\frac{1}{|\mathbf{m}|^{9}}\right) \text {. }
\end{aligned}
$$

\section{B.2. 2 directions unbounded, 1 direction spectral.}

B.2.1. Singular. If $z$ is the spectral direction, the exact Green's function for the continuous problem is

$$
\tilde{G}\left(r, k_{z}\right)= \begin{cases}\frac{1}{2 \pi} \log (r) & \text { if } k_{z}=0 \\ -\frac{1}{2 \pi} K_{0}\left(\left|k_{z}\right| r\right) & \text { otherwise, }\end{cases}
$$

where $r=\sqrt{x^{2}+y^{2}}$, and $K_{0}$ is the modified Bessel function of the second kind. The singular value in $r=0$ is replaced by the cell average value. With the $2 \mathrm{D}$ equivalent radius $r_{e q}=\frac{h^{2}}{\sqrt{\pi}}$,

$$
\tilde{G}\left(0, k_{z}\right)= \begin{cases}\frac{1}{8 \pi}\left(\pi-6+2 \log \left(\frac{\pi}{2} r_{e q}\right)\right) & \text { if } k_{z}=0 \\ -\frac{1-k_{z} r_{e q} K_{1}\left(k_{z} r_{e q}\right)}{\pi\left(k_{z} r_{e q}\right)^{2}} & \text { otherwise }\end{cases}
$$

B.2.2. Regularized. Defining $\rho=\frac{\sqrt{x^{2}+y^{2}}}{\varepsilon}$ and $s_{z}=\varepsilon k_{z}$, the Green's function should be obtained as

$$
\tilde{G}_{m}\left(\rho, s_{z}\right)=-\frac{1}{2 \pi} \int_{0}^{\infty} \frac{\hat{\zeta}_{m}\left(\sqrt{s^{2}+s_{z}^{2}}\right)}{s^{2}+s_{z}^{2}} J_{0}(s \rho) s d s
$$

\footnotetext{
${ }^{5}$ The integration was performed using the quadgk functions of Matlab along with the besseli function.
} 
As noticed in [30], finding a closed form solution to B.11 is not trivial. The authors of the same reference suggest the use of an approximation under the form of a composite expression in the spectral space, which is here reproduced:

$$
\hat{G}_{m}(\mathbf{k}) \approx \begin{cases}\hat{G}_{m}^{2 D}(s) & \text { if } s_{z}=0 \\ -\frac{\hat{\zeta}_{m}\left(\sqrt{s^{2}+s_{z}^{2}}\right)}{|\mathbf{k}|^{2}} & \text { otherwise }\end{cases}
$$

where $s=\varepsilon \sqrt{k_{x}^{2}+k_{y}^{2}} \cdot \hat{G}^{m, 2 D}$ is obtained as the Fourier transform of the solution of a 2D unbounded regularized problem,

$$
G_{m}^{2 D}(\rho)=\frac{1}{2 \pi}\left(\log (\varepsilon \rho)-R_{m}(\rho) e^{-\frac{\rho^{2}}{2}}+\frac{1}{2} E_{1}\left(\frac{\rho^{2}}{2}\right)\right),
$$

with the first exponential integral $E_{1}(z)=\int_{1}^{\infty} \frac{e^{-z q}}{q} d q$, and

$$
R_{2}(\rho)=0, \quad R_{4}(\rho)=\frac{1}{2}, \quad R_{6}(\rho)=\frac{3}{4}-\frac{1}{8} \rho^{2}, \quad R_{8}(\rho)=\frac{11}{12}-\frac{7}{24} \rho^{2}+\frac{1}{48} \rho^{4}, \quad R_{10}(\rho)=\frac{25}{24}-\frac{23}{48} \rho^{2}+\frac{13}{192} \rho^{4}-\frac{1}{384} \rho^{6} .
$$

The limit when $\rho \rightarrow 0$ is obtained as

$$
\begin{gathered}
G_{2}^{2 D}(0)=-\frac{1}{2 \pi}\left(\frac{\gamma}{2}-\log (\sqrt{2} \varepsilon)\right), \quad G_{4}^{2 D}(0)=-\frac{1}{2 \pi}\left(\frac{\gamma}{2}-\log (\sqrt{2} \varepsilon)+\frac{1}{2}\right), \quad G_{6}^{2 D}(0)=-\frac{1}{2 \pi}\left(\frac{\gamma}{2}-\log (\sqrt{2} \varepsilon)+\frac{3}{4}\right), \\
G_{8}^{2 D}(0)=-\frac{1}{2 \pi}\left(\frac{\gamma}{2}-\log (\sqrt{2} \varepsilon)+\frac{11}{12}\right), \quad G_{10}^{2 D}(0)=-\frac{1}{2 \pi}\left(\frac{\gamma}{2}-\log (\sqrt{2} \varepsilon)+\frac{25}{24}\right) .
\end{gathered}
$$

with $\gamma=0.5772156649$ being Euler's constant.

\section{B.3. 1 direction unbounded, 2 directions spectral.}

B.3.1. Singular. If $x$ is the unbounded direction, the exact solution is

$$
\tilde{G}(x, \kappa)= \begin{cases}\frac{1}{2}|x| & \text { if } \kappa=0 \\ -\frac{1}{2} \frac{e^{-\kappa|x|}}{\kappa} & \text { otherwise }\end{cases}
$$

where $\kappa=\sqrt{k_{y}^{2}+k_{z}^{2}}$.

B.3.2. Regularized. Defining $s=\varepsilon \sqrt{k_{y}^{2}+k_{z}^{2}}$ and $\rho=\frac{|x|}{\varepsilon}$, the regularized kernel is

$$
\tilde{G}_{m}(\rho, s)=-\frac{\varepsilon}{4 s}\left(\left(1-\operatorname{erf}\left(\frac{s-\rho}{\sqrt{2}}\right)\right) e^{-s \rho}+\left(1-\operatorname{erf}\left(\frac{s+\rho}{\sqrt{2}}\right)\right) e^{s \rho}\right)-\frac{\varepsilon \sqrt{2}}{\sqrt{\pi}} U_{m}(\rho, s) e^{-\frac{s^{2}+\rho^{2}}{2}}
$$

and

$$
\begin{gathered}
U_{2}(\rho, s)=0, \quad U_{4}(\rho, s)=\frac{1}{4}, \quad U_{6}(\rho, s)=\frac{5}{16}+\frac{1}{16}\left(s^{2}-\rho^{2}\right), \quad U_{8}(\rho, s)=\frac{11}{32}+\frac{1}{12} s^{2}-\frac{1}{8} \rho^{2}-\frac{1}{48} s^{2} \rho^{2}+\frac{1}{96}\left(s^{4}+\rho^{4}\right), \\
U_{10}(\rho, s)=\frac{93}{256}+\frac{73}{768} s^{2}-\frac{47}{256} \rho^{2}-\frac{17}{384} s^{2} \rho^{2}+\frac{11}{768} s^{4}+\frac{23}{768} \rho^{4}+\frac{1}{256}\left(s^{2} \rho^{4}-s^{4} \rho^{2}\right)+\frac{1}{768}\left(s^{6}-\rho^{6}\right) .
\end{gathered}
$$

When $s=0$, the solution of a 1D unbounded problem has to be used,

$$
\tilde{G}_{m}(\rho, 0)=\frac{r}{2} \operatorname{erf}\left(\frac{\rho}{\sqrt{2}}\right)-\frac{\varepsilon}{\sqrt{2 \pi}} V_{m}(\rho),
$$

with

$$
\begin{gathered}
V_{2}(\rho)=\left(1-e^{-\frac{\rho^{2}}{2}}\right), \quad V_{4}(\rho)=\frac{1}{2}\left(1-e^{-\frac{\rho^{2}}{2}}\right), \quad V_{6}(\rho)=\frac{1}{8}\left(3-\left(\rho^{2}+3\right) e^{-\frac{\rho^{2}}{2}}\right), \quad V_{8}(\rho)=\frac{5}{16}-\left(-\frac{1}{48} \rho^{4}+\frac{1}{4} \rho^{2}+\frac{5}{16}\right) e^{-\frac{\rho^{2}}{2}} \\
V_{10}(\rho)=\frac{35}{128}-\left(\frac{1}{384} \rho^{6}-\frac{23}{384} \rho^{4}+\frac{47}{128} \rho^{2}+\frac{35}{128}\right) e^{-\frac{\rho^{2}}{2}}
\end{gathered}
$$

\section{B.4. 3 directions spectral.}


B.4.1. Singular. The well-known exact solution is

$$
\hat{G}(\mathbf{k})=-\frac{1}{k^{2}}
$$

with $k=\sqrt{k_{x}^{2}+k_{y}^{2}+k_{z}^{2}}$. By default, FLUPS arbitrarily sets $\hat{G}(\mathbf{0})=0$.

B.4.2. Regularized. Again with $k=\sqrt{k_{x}^{2}+k_{y}^{2}+k_{z}^{2}}$, the regularized Green's function is given by

$$
\hat{G}_{m}(\mathbf{k})=-\frac{\hat{\zeta}^{m}(\varepsilon k)}{k^{2}}
$$

and again, $\hat{G}^{m}(\mathbf{0})=0$ by default. The limit $m \rightarrow \infty$ corresponds exactly to the singular case.

B.4.3. Lattice Green's Function. The spectral LGF is computed as the spectral equivalent to the finite-difference Poisson equation, $\nabla_{h}^{2} \phi=f$,

$$
\hat{G}_{h}(\mathbf{k})=-\frac{h^{2}}{4 \sin ^{2}\left(k_{x} \frac{h}{2}\right)+4 \sin ^{2}\left(k_{y} \frac{h}{2}\right)+4 \sin ^{2}\left(k_{z} \frac{h}{2}\right)} .
$$

